\title{
Untargeted metabolite profiling on the water-soluble metabolites of edible bird's nest through liquid chromatography-mass spectrometry
}

\author{
Shi-Ruo Tong ${ }^{1}$, Ting-Hun Lee ${ }^{2}$, Soon-Keng Cheong ${ }^{1}$ and Yang-Mooi Lim ${ }^{1,3}$
}

1. Department of Pre-clinical Sciences, Faculty of Medicine and Health Sciences, Universiti Tunku Abdul Rahman, Jalan Sungai Long, Bandar Sungai Long, 43000, Kajang, Cheras, Selangor, Malaysia; 2. Department of Bioprocess and Polymer Engineering, School of Chemical and Energy Engineering, Universiti Teknologi Malaysia, 81310, Johor Bahru, Johor, Malaysia; 3. Centre for Cancer Research, Faculty of Medicine and Health Sciences, Universiti Tunku Abdul Rahman, Jalan Sungai Long, Bandar Sungai Long, 43000, Kajang, Cheras, Selangor, Malaysia.

Corresponding author: Yang-Mooi Lim, e-mail: ymlim@utar.edu.my

Co-authors: SRT: shiruo01670@gmail.com, THL: leetinghun@utm.my, SKC: cheongsk@utar.edu.my Received: 25-09-2019, Accepted: 07-01-2020, Published online: 17-02-2020

doi: www.doi.org/10.14202/vetworld.2020.304-316 How to cite this article: Tong SR, Lee TH, Cheong SK, Lim YM (2020) Untargeted metabolite profiling on the water-soluble metabolites of edible bird's nest through liquid chromatography-mass spectrometry, Veterinary World, 13(2): 304-316.

\begin{abstract}
Background and Aim: Edible bird's nest (EBN) is the nutrient-rich salivary bioproduct produced by swiftlets in Southeast Asia. Currently, researchers are exploring the therapeutic effects of EBN, such as cell growth promotion, antioxidant content, antiviral effects, bone strengthening, eyes care, and neuroprotection bioactivities. The therapeutic effects of EBN have been studied through different extraction methods but the metabolites profile of the EBN in each extract has not yet been elucidated. This study aimed to profile the water-soluble metabolites of EBN prepared in different extraction methods. Subsequently, an extraction method will be selected as an ideal extraction method for untargeted metabolite profiling on the water-soluble metabolites in EBN.
\end{abstract}

Materials and Methods: In this study, water-soluble metabolites of EBN extracted by the four extraction methods were subjected to metabolite profiling through liquid chromatography-mass spectrometry (LC-MS). The extraction methods were acid extraction( $\mathrm{ABN}$ ), pancreatic extraction (EzBN), eHMG extraction, and spray drying of HMG extraction (pHMG). The metabolite profiles, such as the number of metabolites and their identities in each extraction method, were evaluated through LC-MS analysis.

Results: The identity of metabolites present in the four extraction methods is inconsistent. Based on LC-MS analysis, only one and six metabolites were extracted differently through EzBN and ABN, respectively, in the first pre-screening. Through the second LC-MS screening on pHMG and eHMG extraction methods, eHMG was selected as an ideal extraction method due to the highest numbers of water-soluble metabolites with an amount of 193 was detected. Besides, eHMG extraction method was able to extract sialic acid and a high percentage of secondary metabolites.

Conclusion: This study suggests that eHMG is the ideal extraction method for extracting higher number of water-soluble metabolites from EBN and could be further developed as an extraction method for industry application. In addition, this study also has identified the types of primary and secondary metabolites present in EBN.

Keywords: edible bird's nest, extraction method, liquid chromatography-mass spectrometry, untargeted metabolite profiling.

\section{Introduction}

Edible bird's nest (EBN) is a well-known bioproduct made from the saliva secretion of swiftlet, specifically from the two genera of Aerodramus and Collocalia. The swiftlet from the two genera is mostly habitat in Southeast Asia $[1,2]$. The main constituents of EBN are proteins, carbohydrates, lipids, and a group of minerals such as calcium, sodium, potassium, magnesium, phosphorus, iron, zinc, copper, chromium, and selenium [2-5]. EBN has been regarded as traditional Chinese medicine by the practitioners in Qing dynasty due to its recuperative

Copyright: Tong, et al. Open Access. This article is distributed under the terms of the Creative Commons Attribution 4.0 International License (http://creativecommons.org/licenses/by/4.0/), which permits unrestricted use, distribution, and reproduction in any medium, provided you give appropriate credit to the original author(s) and the source, provide a link to the Creative Commons license, and indicate if changes were made. The Creative Commons Public Domain Dedication waiver (http://creativecommons.org/ publicdomain/zero/1.0/) applies to the data made available in this article, unless otherwise stated. properties $[1,6]$. The recuperative properties of EBN are highlighted with the effect of boosting immune system, treating malnutrition, improving metabolism, enhancing skin complexion and alleviating asthma, helping in phlegm clearance, relieving cough, nourishing children, libido raising, enhancing renal function, recovery from illness and surgery, as well as improving concentration [7]. Recently, EBN is further demonstrated for its properties on suppressing the virus, inflammation and oxidative stress, strengthening bone, eye caring, and neuroprotective properties [8-14]. On the other hand, Roh et al. [15] and Kong et al. [16] have reported the proliferative effects of EBN on human adipose-derived stem cells and normal human fibroblasts with the presence of epidermal growth factor-like activity. In summary, EBN acts as a dual function bioproduct with both its nutritional and therapeutic values.

To study the constituents of EBN and its therapeutic effects, the development of an ideal extraction 
methodology of EBN is very important. Several extraction methodologies were developed and used for studying the bioactivities of EBN. The study by Guo et al. [9] documented strong inhibition of influenza viruses by EBN extract that is pre-treated with pancreatin. Besides, Abidin et al. [11] also reported that the EBN extract prepared by eHMG extraction method successfully stimulated and enhanced the proliferation of corneal keratocytes in wound healing without altering their functionality. Chua et al. [17] prepared EBN extracts by the water extraction method (HMG). These extracts exhibited strong chondroprotective effects on osteoarthritis (OA). In addition, Aswir and Wan Nazaimoon [18] have documented acid-extracted EBN exhibited an anti-inflammation effect by significantly reducing the production of the inflammatory protein, tumor necrosis factor-alpha. In view of all the works, it is observed that different EBN extract obtained through different extraction methods showed different therapeutic effects. One possible explanation is because the extraction of an active component is highly dependent on the extraction method employed. Thus far, the identity of the metabolites in each of these extractions has not yet been further studied for the underlying mechanism of actions for their therapeutic effects. Hence, future study could be carried out to confirm the therapeutic effects of the metabolites.

Metabolite profiling is a powerful scientific tool for a complete investigation of a group of small molecules. This approach often used in analyzing biological components for the identification of potential biomarkers for certain diseases [19]. Recently, metabolite profiling has gained fame in food classification [20,21]; this is due to its untargeted analysis approach with the potential to cover the whole or the maximum metabolomics molecular information of foods. One of the examples of using the metabolite profiling approach on EBN has successfully demonstrated in the study done by Chua et al. [22]. The metabolites of the EBN were extracted through the chloroform/methanol solvent extraction, which was then successfully identified through gas chromatography-mass spectrometry (MS) and liquid chromatography-MS (LC-MS) techniques.

Since water is commonly used to prepare EBN essence for consumption and the metabolites of EBN are not fully established yet, this study aimed to preliminary profile the water-soluble metabolites of EBN prepared in different extraction methods. Subsequently, an extraction method will be selected as an ideal extraction method for untargeted metabolite profiling on the water-soluble metabolites in EBN.

\section{Materials and Methods}

Ethical approval

The study did not involve any live animals, so no ethical approval was required.

\section{Chemicals}

LC-MS grade formic acid and acetonitrile were purchased from Fisher Scientific (Waltham, MA,
USA). Deionized water was obtained from a Barnstead GenPure water purification system (Thermo Fisher Scientific Inc., Waltham, MA, USA).

\section{Sample collection, preparation, and extraction}

Raw unclean EBNs samples were collected collectively from different swiftlet premises located in Johor, Malaysia. The feathers and impurities were manually removed with forceps, and the raw unclean EBN was ground with mortar and pestle. Ground EBN was sieved through a $0.4 \mathrm{~mm}$ wire mesh to further separate the smaller pieces of feathers and impurities. The unclean EBN powder was then placed in an air force oven at $50-55^{\circ} \mathrm{C}$ overnight to reduce the moisture content.

There were four extraction methods selected for the comparison in this study, namely, eHMG, pHMG, $\mathrm{ABN}$, and EzBN extraction methods. The raw unclean EBN was extracted with the proprietary methods of eHMG [11] and pHMG (the spray-dried of HMG extract) [17] that were innovated and standardized by School of Chemical and Energy Engineering in Universiti Teknologi Malaysia (UTM). These methods were modified based on the methods presented by Oda et al. [23] and Goh et al. [24]. Besides, another acid extraction $(\mathrm{ABN})$ and pancreatin extraction (EzBN) were developed by the team of Universiti Tunku Abdul Rahman (UTAR) in 2016 [25] with some modification from the methods presented by Aswir and Wan Nazaimoon [18] and Goh et al. [9].

\section{eHMG and $p H M G$}

Due to the proprietary issue on these two extraction methods, the details of these two methods were unable to be described in this report.

\section{Acid extraction ( $A B N)$}

The EBN powder was suspended in deionized water at $0.2 \%(\mathrm{w} / \mathrm{v})$ and left for $24 \mathrm{~h}$. The mixture was then boiled at $80^{\circ} \mathrm{C}$ with $2 \%(\mathrm{v} / \mathrm{v})$ of $0.4 \mathrm{M}$ sulfuric acid for $4 \mathrm{~h}$. The extract was allowed to cool down and centrifuged at $2716 \mathrm{~g}(5000 \mathrm{rpm})$ for $15 \mathrm{~min}$. The $\mathrm{pH}$ of the supernatant collected was neutralized to $\mathrm{pH}$ 7.0. The white precipitated formed was removed through centrifugation with $2716 \mathrm{~g}(5000 \mathrm{rpm})$ for $15 \mathrm{~min}$ at $4^{\circ} \mathrm{C}$. The supernatant was collected and kept at $4^{\circ} \mathrm{C}$ for further analysis.

\section{Pancreatin extraction ( $E z B N)$}

The EBN powder was suspended in deionized water at $0.2 \%(\mathrm{w} / \mathrm{v})$ and left for $24 \mathrm{~h}$. The EBN mixture was boiled at $100^{\circ} \mathrm{C}$ for $30 \mathrm{~min}$. An amount of $1 \mathrm{ml}$ of $0.5 \mathrm{mg} / \mathrm{ml}$ pancreatin was added into EBN mixture and was allowed for the reaction at $45^{\circ} \mathrm{C}$ for $4 \mathrm{~h}$ with $\mathrm{pH}$ 8.5-9.0. The enzyme was inactivated by heating at $90^{\circ} \mathrm{C}$ for $10 \mathrm{~min}$. The supernatant was collected after centrifugation at $2716 \mathrm{~g}(5000 \mathrm{rpm})$ for $15 \mathrm{~min}$. The extract was kept at $4^{\circ} \mathrm{C}$.

Before subjecting the extracts to LC-MS analysis, all the four extracts were centrifuged at $9660 \mathrm{~g}$ 
$(12,000 \mathrm{rpm})$ for $10 \mathrm{~min}$ and the supernatant of the extracts was filtered through $0.2 \mu \mathrm{m}$ polytetrafluoroethylene membranes.

\section{Quadrupole time-of-flight (QTOF) LC-MS analysis}

The four EBN extracts were qualitatively analyzed using Agilent 6560 Ion Mobility QTOF (IM-QTOF) LC-MS system that coupled with the Agilent 1290 ultra-high-performance liquid chromatography (Agilent Technologies, USA). The metabolites present in the EBN extracts were separated through POROSHELL 120 EC-C18 (4.6×100 mm; $2.7 \mu$; Agilent Technologies, USA) chromatographic column with the mobile phase that consisted of (A) $0.1 \%$ formic acid in water and (B) $0.1 \%$ formic acid in acetonitrile. All the four EBN extracts were undergone the first pre-screening evaluation with the elution of 5-95\% B (0.0-1.0 min) and 95-5\% B (1.0$15.0 \mathrm{~min})$. The flow rate was set at $1.0 \mathrm{ml} / \mathrm{min}$. The two extracts with a higher number of metabolites were selected and further subjected for the second LC-MS screening with modified mobile phase elution. The condition of the modified elution was set as follows: 5\% B (0.0-2.0 min), 5-15\% B (2.0-4.0 min), 15-25\% B (4.0-6.0 min), 25-35\% B (6.0-8.0 min), 35-45\% B (8.0-10.0 $\mathrm{min}), 45-50 \% \mathrm{~B}(10.0-12.0 \mathrm{~min}), 50-75 \% \mathrm{~B}$ (12.0-16.0 $\mathrm{min}), 75-100 \% \mathrm{~B}$ (16.0-20.0 $\mathrm{min}), 100-5 \%$ $\mathrm{B}(20.0-20.1 \mathrm{~min})$, and isocratic at 5\% (20.1-25 $\mathrm{min})$. The flow rate was modified to $0.3 \mathrm{ml} / \mathrm{min}$.

The other setting parameters for IM-TOF analysis remained the same throughout the analysis process. The injection volume was $1 \mu l$ and the column temperature was maintained at $40^{\circ} \mathrm{C}$. The acquisition of the metabolites was performed in positive (ES+) mode. The mass spectra were recorded over an $\mathrm{m} / \mathrm{z}$ range from 100 to 1000 . Deionized water was used as the background blank. Whereas, the operating conditions of the mass spectrometer were set as follows: Capillary voltage of $4000 \mathrm{~V}$, nozzle voltage of $500 \mathrm{~V}$, and fragmentor voltage of $365 \mathrm{~V}$ were maintained. Nebulizer pressure $\left(\mathrm{N}_{2}\right)$ was kept at 20 psi, drying gas temperature was maintained at $225^{\circ} \mathrm{C}$. Drying gas flow was $13 \mathrm{~L} / \mathrm{min}$ and sheath gas flow was $12 \mathrm{~L} / \mathrm{min}$ at $400^{\circ} \mathrm{C}$.

\section{Data mining and metabolites identification}

The metabolite features from the acquired MS spectral raw data were extracted with the untargeted molecular feature extraction algorithm in Agilent MassHunter Workstation - Qualitative Analysis software B.07.00 (Agilent Technologies, USA). The algorithm filtered off the peak height with 100 counts to avoid the noise spectral picking, as well as the mass of internal reference ions with 121.0967 and 922.1389. Then, the algorithm locates the covariant ions in the chromatogram and grouped them as a single metabolite feature using the information of mass, isotopic distribution with common organic elements $(\mathrm{C}, \mathrm{H}, \mathrm{O}$, $\mathrm{N}, \mathrm{P}, \mathrm{Cl}, \mathrm{F}$, and S), charge-state and adducts of sodium, potassium, and ammonium. The extracted metabolite features were characterized by retention time (RT) and intensity.

The identity of the extracted metabolite features was searched against METLIN Personal Metabolite Database in the MassHunter software based on the accurate mass and RT (optional). The mass and RT tolerance of the compound identity matching was restricted to $\pm 5 \mathrm{ppm}$ and $\pm 0.1 \mathrm{~min}$ (optional), respectively. The accuracy of the identity of each metabolite was calculated as a score. The metabolites list of each extract was retained if the identity of the metabolite fulfilled the threshold score of 80 , and the error of database matching was less than \pm 5 ppm.

\section{Results and Discussion}

\section{The efficiency of EBN extraction methods}

The method of extraction is a crucial process that maximizes the extraction of the bioactive metabolites from EBN. To search for an ideal extraction method for the untargeted metabolite profiling of EBN, four different extraction methods with the therapeutic effects were assessed and evaluated. For example, pancreatin extraction with antiviral effect as reported by Guo et al. [9]; eHMG extraction with the effect of enhancing proliferation of corneal keratocytes by Abidin et al. [11]; HMG extraction showed chondroprotective effect on OA as documented by Chua et al. [17]; and finally the acid extraction with anti-inflammation bioactivities reported by Aswir and Wan Nazaimoon [18]. The approach of LC-MS is recognized with its high sensitivity, accuracy, and reproducibility [26-28]; thus, there was no technical replicate done in this untargeted metabolite profiling analysis.

The number of detected metabolites in each of the extraction method was analyzed by MassHunter software. Nearly $37-67 \%$ out of the total metabolites from the four different extracts were putatively identified by matching with the METLIN metabolites database. The complete information of all the identified metabolites in each extraction method is detailed in Table-1. The identities of the extracted metabolites are unique among the four different extracts, suggesting that there is no single extraction method that could extract all types of metabolites due to the differences in natural physicochemical properties of the metabolites [29-32].

Based on the mobile phase for compound separation in the first screening evaluation, there were significant differences in the number of extracted metabolites under each extraction method (Table-2a). The highest total number of metabolites obtained was from $\mathrm{pHMG}$ extract and followed by eHMG extract. The total number of metabolites detected in both of pHMG and eHMG extracts was greater than EzBN and $\mathrm{ABN}$ extracts, with approximately 20-30 times and 4-5 times, respectively. However, the LC-MS separation for each extract was not well defined by referring to the chromatograms obtained (Figure-1). Therefore, the second screening evaluation was carried out with an improved LC-MS mobile phase. 
Table-1: Information of the metabolites in each extracts with first pre-screening by QTOF LC-MS.

\begin{tabular}{|c|c|c|c|c|c|c|c|c|}
\hline Number & RT (min) & Ion & Mass & $\mathbf{m} / \mathbf{z}$ & $\begin{array}{l}\text { Molecular } \\
\text { formula }\end{array}$ & Score & $\begin{array}{l}\text { DB differences } \\
(\mathrm{ppm})\end{array}$ & $\begin{array}{l}\text { Putatively identified } \\
\text { metabolites }\end{array}$ \\
\hline \multicolumn{9}{|c|}{ ABN } \\
\hline 1 & 1.001 & $\left(\mathrm{M}+\mathrm{NH}_{4}\right)^{+}$ & 104.0375 & 122.0713 & $\mathrm{C}_{6} \mathrm{H}_{4} \mathrm{~N}_{2}$ & 85.65 & -0.48 & 4-Cyanopyridine \\
\hline 2 & 1.005 & $(\mathrm{M}+\mathrm{H})^{+}$ & 273.1082 & 274.1154 & $\mathrm{C}_{9} \mathrm{H}_{15} \mathrm{~N}_{5} \mathrm{O}_{5}$ & 97.11 & -3.06 & 4a-Peroxy-tetrahydrobiopterin \\
\hline 3 & 1.005 & $(\mathrm{M}+\mathrm{H})^{+}$ & 291.1206 & 292.1278 & $\mathrm{C}_{14} \mathrm{H}_{17} \mathrm{~N}_{3} \mathrm{O}_{4}^{3}$ & 89.12 & 4.47 & Serinyl-Tryptophan \\
\hline 4 & 1.005 & $(\mathrm{M}+\mathrm{H})^{+}$ & 309.1332 & 310.1406 & $\mathrm{C}_{17}^{14} \mathrm{H}_{18} \mathrm{~F}_{3}^{3} \mathrm{NO}$ & 87.45 & 2.62 & Fluoxetine \\
\hline 5 & 1.039 & $(\mathrm{M}+\mathrm{Na})^{+}$ & 325.0796 & 348.0687 & $\mathrm{C}_{14} \mathrm{H}_{15} \mathrm{NO}_{8}$ & 84.49 & 0.49 & Pancratistatin \\
\hline 6 & 1.083 & $(\mathrm{M}+\mathrm{Na})^{+}$ & 291.0943 & 314.0835 & $\mathrm{C}_{11}^{14} \mathrm{H}_{17} \mathrm{NO}_{8}^{\circ}$ & 95.46 & 3.83 & $\begin{array}{l}\text { 2-Deoxy-2,3-dehydro-N- } \\
\text { acetylneuraminic acid }\end{array}$ \\
\hline \multicolumn{9}{|c|}{ EzBN } \\
\hline 1 & 1.502 & $(\mathrm{M}+\mathrm{H})^{+}$ & 109.0643 & 110.0715 & $\mathrm{C}_{5} \mathrm{H}_{7} \mathrm{~N}_{3}$ & 85.25 & -2.46 & 2-Aminomethylpyrimidine \\
\hline \multicolumn{9}{|c|}{ eHMG } \\
\hline 1 & 0.741 & $(\mathrm{M}+\mathrm{H})^{+}$ & 379.1125 & 380.1197 & $\mathrm{C}_{14} \mathrm{H}_{21} \mathrm{NO}_{11}$ & 83.05 & -2.62 & Chondroitin \\
\hline 2 & 0.924 & $(\mathrm{M}+\mathrm{Na})^{+}$ & 333.1523 & 356.1415 & $\mathrm{C}_{13}^{14} \mathrm{H}_{23} \mathrm{~N}_{3} \mathrm{O}_{7}$ & 80.80 & 3.82 & Ser Asp Leu \\
\hline 3 & 1.007 & $(\mathrm{M}+\mathrm{H})^{+}$ & 385.2081 & 386.2152 & $\mathrm{C}_{15}^{13} \mathrm{H}_{27}^{23} \mathrm{~N}_{7}^{3} \mathrm{O}_{5}$ & 83.05 & -1.87 & Asn Pro Arg \\
\hline 4 & 1.042 & $(\mathrm{M}+\mathrm{H})^{+}$ & 311.1692 & 312.1764 & $\mathrm{C}_{12}^{15} \mathrm{H}_{21} \mathrm{~N}_{7} \mathrm{O}_{3}^{5}$ & 91.60 & 4.34 & Arginyl-Histidine \\
\hline 5 & 1.112 & $(\mathrm{M}+\mathrm{H})^{+}$ & 344.2172 & 345.2244 & $\mathrm{C}_{14}^{12} \mathrm{H}_{28} \mathrm{~N}_{6} \mathrm{O}_{4}^{3}$ & 84.88 & 0.07 & Gly Ile Arg \\
\hline 6 & 1.138 & $\left(\mathrm{M}+\mathrm{NH}_{4}\right)^{+}$ & 384.2144 & 402.2484 & $\mathrm{C}_{20} \mathrm{H}_{32} \mathrm{O}_{7}$ & 81.25 & 1.10 & Cinnzeylanol \\
\hline 7 & 1.311 & $(\mathrm{M}+\mathrm{H})^{+}$ & 387.2238 & 388.2313 & $\mathrm{C}_{15} \mathrm{H}_{29} \mathrm{~N}_{7} \mathrm{O}_{5}$ & 94.39 & -2.03 & Arg Asn Val \\
\hline 8 & 1.383 & $(\mathrm{M}+\mathrm{H})^{+}$ & 373.2333 & 374.2405 & $\mathrm{C}_{16} \mathrm{H}_{31} \mathrm{~N}_{5} \mathrm{O}_{5}$ & 81.27 & -2.19 & Lys Asn Leu \\
\hline 9 & 4.299 & $(\mathrm{M}+\mathrm{H})^{+}$ & 654.3986 & 655.4060 & $\mathrm{C}_{35} \mathrm{H}_{58} \mathrm{O}_{11}$ & 93.38 & -1.00 & Filipin III \\
\hline 10 & 4.316 & $(\mathrm{M}+\mathrm{H})^{+}$ & 130.0741 & 131.0813 & $\mathrm{C}_{5} \mathrm{H}_{10} \mathrm{~N}_{2} \mathrm{O}_{2}$ & 87.43 & 1.25 & $\begin{array}{l}\text { L-cis-3-Amino-2- } \\
\text { pyrrolidinecarboxylic acid }\end{array}$ \\
\hline 11 & 4.342 & $(\mathrm{M}+\mathrm{H})^{+}$ & 114.0433 & 115.0505 & $\mathrm{C}_{4} \mathrm{H}_{6} \mathrm{~N}_{2} \mathrm{O}_{2}$ & 86.61 & -3.03 & Muscimol \\
\hline \multicolumn{9}{|c|}{ pHMG } \\
\hline 1 & 1.019 & $(\mathrm{M}+\mathrm{H})^{+}$ & 166.0270 & 167.0344 & $\mathrm{C}_{8} \mathrm{H}_{6} \mathrm{O}_{4}$ & 85.71 & -2.59 & 3-Formylsalicylic acid \\
\hline 2 & 1.020 & $(\mathrm{M}+\mathrm{Na})^{+}$ & 383.1430 & 406.1321 & $\mathrm{C}_{14} \mathrm{H}_{25} \mathrm{NO}_{11}$ & 98.38 & -0.64 & Lacto-N-biose I \\
\hline 3 & 1.022 & $(\mathrm{M}+\mathrm{Na})^{+}$ & 309.1065 & 332.0956 & $\mathrm{C}_{11}^{14} \mathrm{H}_{19}^{25} \mathrm{NO}_{9}^{11}$ & 82.57 & -1.76 & N-Acetyl-b-neuraminic acid \\
\hline 4 & 1.023 & $(\mathrm{M}+\mathrm{Na})^{+}$ & 325.0792 & 348.0685 & $\mathrm{C}_{14}^{11} \mathrm{H}_{15} \mathrm{NO}_{8}^{9}$ & 82.22 & 1.85 & Pancratistatin \\
\hline 5 & 1.024 & $(\mathrm{M}+\mathrm{H})^{+}$ & 203.0798 & 204.0870 & $\mathrm{C}_{8}^{14} \mathrm{H}_{13} \mathrm{NO}_{5}^{8}$ & 86.39 & -1.97 & N2-Acetyl-L-aminoadipate \\
\hline 6 & 1.026 & $(\mathrm{M}+\mathrm{Na})^{+}$ & 291.0960 & 314.0851 & $\mathrm{C}_{12}^{0} \mathrm{H}_{13} \mathrm{~N}_{5} \mathrm{O}_{4}$ & 84.24 & 2.72 & Toyocamycin \\
\hline 7 & 1.075 & $(\mathrm{M}+\mathrm{Na})^{+}$ & 291.0954 & 314.0847 & $\mathrm{C}_{11}^{12} \mathrm{H}_{17} \mathrm{NO}_{8}$ & 83.71 & 0.12 & $\begin{array}{l}\text { 2,7-Anhydro-alpha-N- } \\
\text { acetylneuraminic acid }\end{array}$ \\
\hline 8 & 1.314 & $(\mathrm{M}+\mathrm{H})^{+}$ & 137.0478 & 138.0551 & $\mathrm{C}_{7} \mathrm{H}_{7} \mathrm{NO}_{2}$ & 87.40 & -1.17 & 2-Pyridylacetic acid \\
\hline 9 & 1.319 & $(\mathrm{M}+\mathrm{Na})^{+}$ & 145.0770 & 168.0660 & $\mathrm{C}_{9} \mathrm{H}_{9} \mathrm{~N}_{2}$ & 81.66 & -2.63 & 4-Aminomethylindole \\
\hline 10 & 1.402 & $(\mathrm{M}+\mathrm{H})^{+}$ & 245.1385 & 246.1457 & $\mathrm{C}_{10} \mathrm{H}_{19} \mathrm{~N}_{3} \mathrm{O}_{4}$ & 81.33 & -3.66 & Asn Leu \\
\hline 11 & 1.514 & $(\mathrm{M}+\mathrm{H})^{+}$ & 135.0544 & 136.0617 & $\mathrm{C}_{5} \mathrm{H}_{5} \mathrm{~N}_{5}$ & 84.12 & 0.94 & Adenine \\
\hline 12 & 1.542 & $\left(\mathrm{M}+\mathrm{NH}_{4}\right)^{+}$ & 256.0582 & 274.0920 & $\mathrm{C}_{11} \mathrm{H}_{12} \mathrm{O}_{7}$ & 93.89 & 0.53 & Piscidic Acid \\
\hline 13 & 5.692 & $(\mathrm{M}+\mathrm{H})^{+}$ & 101.0840 & 102.0912 & $\mathrm{C}_{5} \mathrm{H} 1_{1} \mathrm{NO}$ & 87.59 & 0.67 & $\begin{array}{l}\text { 2-Methylpropanal } \\
\text { O-methyloxime }\end{array}$ \\
\hline
\end{tabular}

RT=Retention time, DB=Database, LC-MS=Liquid chromatography-mass spectrometry, QTOF=Quadrupole time-of-flight

Table-2: Number of metabolites detected and identified by QTOF LC-MS in each of the extracts for the first prescreening and the second screening with the optimized LC-MS parameters.

\begin{tabular}{lccc}
\hline Extracts & Total metabolites & Putatively identified metabolites & Metabolites after filtering* \\
\hline (a) First pre-screening & 18 & 7 & 6 \\
ABN & 3 & 2 & 1 \\
EzBN & 69 & 26 & 11 \\
eHMG** & 85 & 34 & 13 \\
pHMG** & 775 & 468 & 193 \\
(b) Second screening with optimized parameters & 96 & 42 \\
eHMG & 168 & & \\
pHMG & & & \\
\hline
\end{tabular}

*Metabolites filtering is based on the presence of contaminants, the score and database matching error (ppm). $* * T h e$ extracts were selected for the second screening with the optimized LC-MS parameters. LC-MS=Liquid chromatographymass spectrometry, QTOF=Quadrupole time-of-flight

Both of the eHMG and pHMG extraction methods were selected to undergo the second screening evaluation since they showed greater efficacy in extracting the higher number of metabolites from EBN in the first screening evaluation.
The second screening evaluation with an optimized LC-MS mobile phase for separating compounds has greatly improved the elution efficacy and increased the number of analyzed metabolites (Figure-2a and b). The good separation in the liquid 


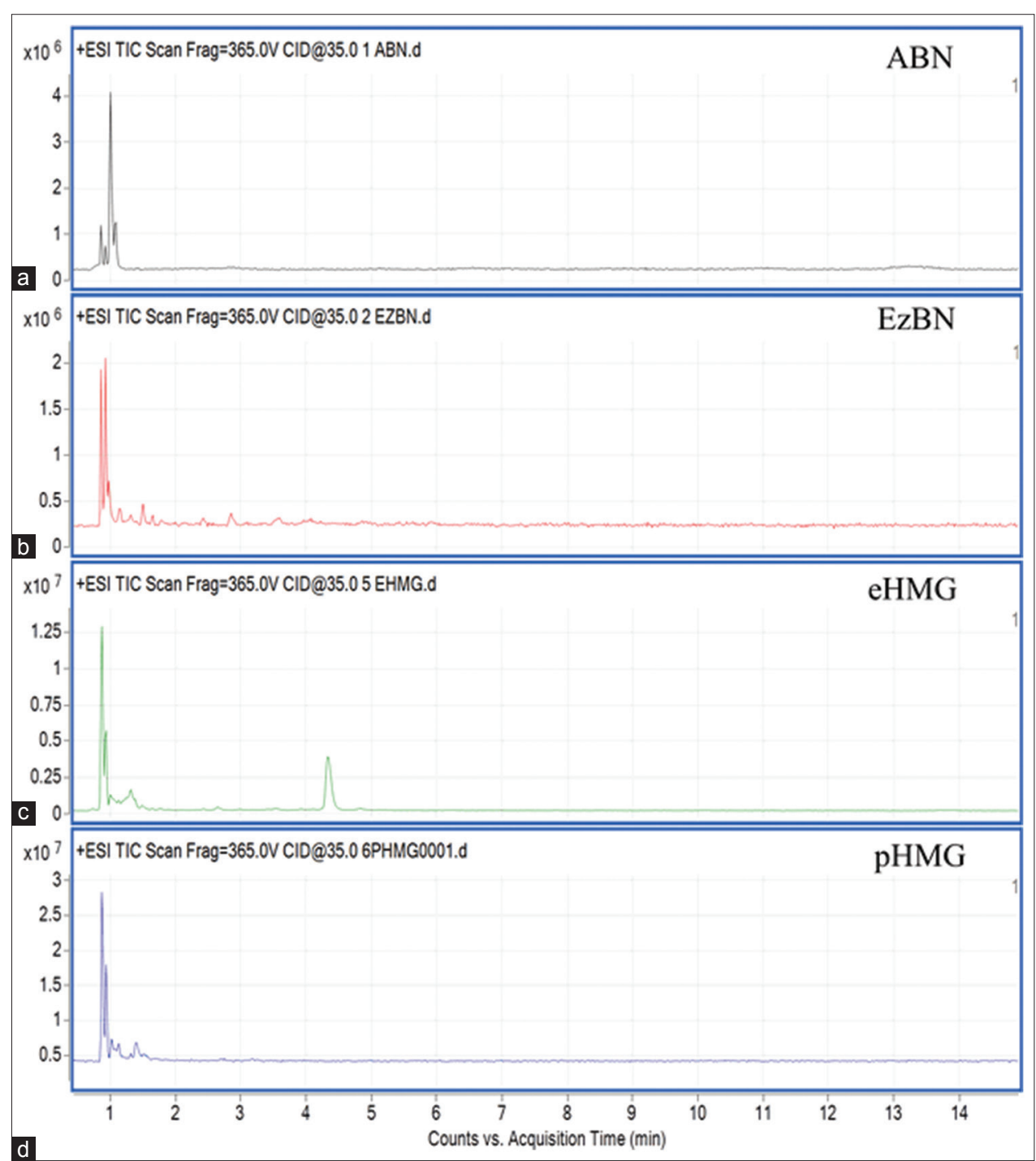

Figure-1: Total ion chromatograms of the first liquid chromatography/mass spectrometry (LC-MS) pre-screening on edible bird's nest extraction methods (a) ABN, (b) EzBN, (c) eHMG, and (d) pHMG. The LC-MS chromatograms are obtained from $\mathrm{ES}+$ mode.

chromatography has broadened the range of eluted metabolites. Hence, the second screening evaluation has provided a better comparison between the eHMG and pHMG extraction methods. The eHMG extraction method has successfully recovered a significant number in total extracted metabolites as compared with pHMG (Table-2b). There were 193 metabolites detected from eHMG extraction method (Table-2b), which are more than 26 non-polar metabolites detected in the study done by Chua et al. [22]. Therefore, the eHMG extraction method was selected as the ideal extraction method because it provided the maximal recovery of the number of water-soluble metabolites present in EBN.

\section{The metabolite profile of extraction methods}

In the second screening evaluation, there were approximately more than half out of the total metabolites $(60.39 \%$ and $57.14 \%$ of metabolites, respectively) from eHMG and pHMG extracts that were putatively identified. The information of the retained metabolites for both eHMG and $\mathrm{pHMG}$ extraction methods in the second screening evaluation are shown in Tables-3 and 4, respectively. Based on the comparison between eHMG and pHMG extraction methods in the second screening evaluation, 24 out of the total identified metabolites were found to be similar in each extract (Figure-2c). The result indicated that the eHMG extraction method not only extracted a greater number of metabolites but also there were approximately $57.14 \%$ of the metabolites from pHMG extraction method which were found to be similar to eHMG. The identities of the metabolites that found to be similar in both of the extraction methods are marked in Tables-3 and 4.

Sialic acid is known as the key component of EBN because it is served as the unique quantitative marker for grading the EBN. In this study, sialic acid was identified in the eHMG extraction method with the identity of 2,7-Anhydro-alpha-N-acetylneuraminic acid (Table-3). The result agreed with the previous studies that $\mathrm{N}$-acetylneuraminic acid (NANA) is the predominant form of sialic acid in EBN [33-35]. The 


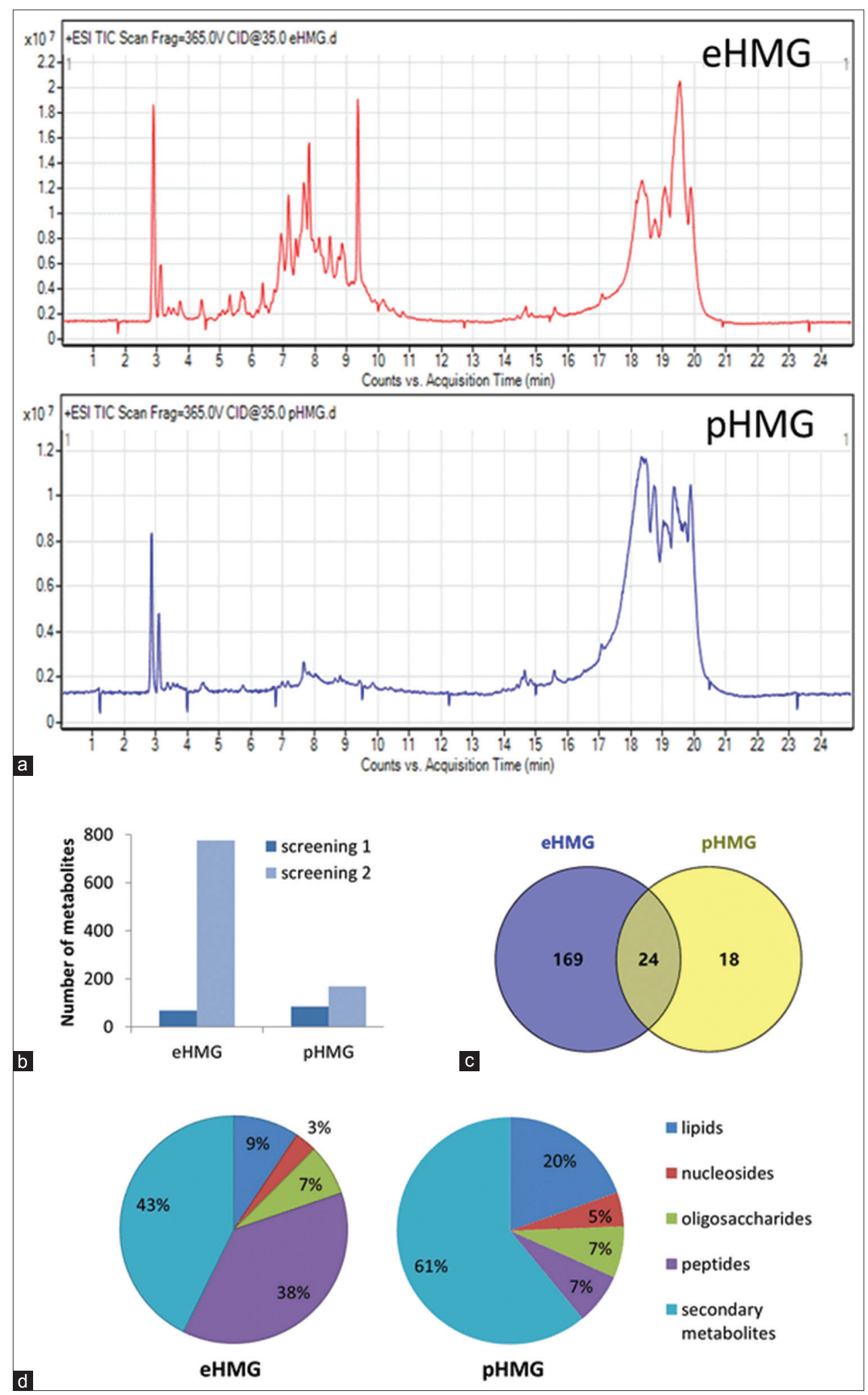

Figure-2: The second liquid chromatography/mass spectrometry (LC-MS) screening with optimized parameters on eHMG and pHMG extracts. (a) Total ion chromatograms of eHMG and pHMG extraction methods selected from the first prescreening. The LC-MS chromatograms were obtained from ES+mode. (b) The efficiency comparison between the first and second screening for both eHMG and pHMG extracts. (c) The number of metabolites that found similar between pHMG and eHMG extracts. The comparison was made based on the identified metabolites that the contaminant was filtered off. (d) The classification of edible bird's nest metabolites in eHMG and pHMG extracts. The classification was based on the metabolite identities after removing the contaminants.

detected of sialic acid in eHMG extract has further convinced that eHMG extraction method is more suitable as the ideal extraction method.
The type of metabolites present in eHMG and pHMG extracts (from the second screening) was further categorized into five groups based on the 
Table-3: Information of the metabolites in eHMG extract. The metabolites are identified by QTOF LC-MS with second screening evaluation.

\begin{tabular}{|c|c|c|c|c|c|c|c|c|}
\hline Number & $\begin{array}{c}\text { RT } \\
(\mathbf{m i n})\end{array}$ & Ion & Mass & $\mathbf{m} / \mathbf{z}$ & $\begin{array}{l}\text { Molecular } \\
\text { formula }\end{array}$ & $\begin{array}{l}\text { Score } \\
\text { (DB) }\end{array}$ & $\begin{array}{l}\text { DB differences } \\
(\mathrm{ppm})\end{array}$ & $\begin{array}{l}\text { Putatively identified } \\
\text { metabolites }\end{array}$ \\
\hline 1 & 7.678 & $\left(\mathrm{M}+\mathrm{NH}_{4}\right)^{+}$ & 188.1052 & 206.1388 & $\mathrm{C}_{9} \mathrm{H}_{16} \mathrm{O}_{4}$ & 87.23 & -1.78 & $\begin{array}{l}(+/-) \text {-Ethyl } \\
\text { 3-acetoxy-2-methylbutyrate }\end{array}$ \\
\hline 2 & 6.351 & $(\mathrm{M}+\mathrm{H})^{+}$ & 156.0533 & 157.0605 & $\mathrm{C}_{6} \mathrm{H}_{8} \mathrm{~N}_{2} \mathrm{O}_{3}$ & 86.21 & 1.11 & (S)-3-(Imidazol-5-yl)lactate \\
\hline 3 & 9.285 & $\left(\mathrm{M}+\mathrm{NH}_{4}\right)^{+}$ & 146.0481 & 164.0819 & $\mathrm{C}_{8} \mathrm{H}_{6} \mathrm{~N}_{2} \mathrm{O}$ & 87.68 & -0.82 & $1(2 \mathrm{H})$-Phthalazinone \\
\hline 4 & 7.814 & $(\mathrm{M}+\mathrm{H})^{+}$ & 225.1118 & 226.1190 & $\mathrm{C}_{10} \mathrm{H}_{15} \mathrm{~N}_{3} \mathrm{O}_{3}$ & 85.52 & -1.99 & $\begin{array}{l}\text { 1-(Methylnitrosoamino)-4- } \\
\text { (3-pyridinyl)-1,4-butanediol }\end{array}$ \\
\hline 5 & 7.154 & $\left(\mathrm{M}+\mathrm{NH}_{4}\right)^{+}$ & 151.1003 & 169.1341 & $\mathrm{C}_{9} \mathrm{H}_{13} \mathrm{NO}$ & 85.35 & -3.75 & $\begin{array}{l}\text { 1,2,3,4,5,6-Hexahydro-5- } \\
\text { methyl-7H-cyclopenta[b] } \\
\text { pyridin-7-one }\end{array}$ \\
\hline 6 & 19.916 & $(\mathrm{M}+\mathrm{H})^{+}$ & 310.2875 & 311.2947 & $\mathrm{C}_{20} \mathrm{H}_{38} \mathrm{O}_{2}$ & 82.62 & -1.10 & 15Z-eicosenoic acid \\
\hline 7 & 19.852 & $\left(\mathrm{M}+\mathrm{NH}_{4}\right)^{+}$ & 168.1882 & 186.2220 & $\mathrm{C}_{12}^{20} \mathrm{H}_{24}^{38}$ & 93.33 & -2.37 & 1-Dodecene* \\
\hline 8 & 17.086 & $(\mathrm{M}+\mathrm{H})^{+}$ & 203.0811 & 204.0882 & $\mathrm{C}_{9} \mathrm{H}_{17}^{24} \mathrm{NS}_{2}$ & 90.55 & -4.19 & $\begin{array}{l}\text { 1-Isothiocyanato-7- } \\
\text { (methylthio)heptane* }\end{array}$ \\
\hline 9 & 13.104 & $(\mathrm{M}+\mathrm{H})^{+}$ & 115.0456 & 116.0528 & $\mathrm{C}_{5} \mathrm{H}_{9} \mathrm{NS}$ & 95.32 & -0.49 & 1-Isothiocyanatobutane* \\
\hline 10 & 19.913 & $\left(\mathrm{M}+\mathrm{NH}_{4}\right)^{+}$ & 392.4382 & 410.4720 & $\mathrm{C}_{28}^{5} \mathrm{H}_{56}$ & 95.74 & 0.02 & 1-Octacosene* \\
\hline 11 & 8.497 & $(\mathrm{M}+\mathrm{Na})^{+}$ & 303.1824 & 326.1717 & $\mathrm{C}_{18}^{28} \mathrm{H}_{25}^{56} \mathrm{NO}_{3}$ & 93.54 & 3.33 & 1-O-Desmethyltetrabenazine \\
\hline 12 & 6.266 & $(\mathrm{M}+\mathrm{H})^{+}$ & 129.0429 & 130.0503 & $\mathrm{C}_{5} \mathrm{H}_{7} \mathrm{NO}_{3}$ & 94.98 & -2.57 & $\begin{array}{l}\text { 1-Pyrroline-4-hydroxy-2- } \\
\text { carboxylate }\end{array}$ \\
\hline 13 & 7.633 & $(\mathrm{M}+\mathrm{H})^{+}$ & 365.1324 & 366.1397 & $\mathrm{C}_{14} \mathrm{H}_{23} \mathrm{NO}_{10}$ & 98.61 & -0.58 & $\begin{array}{l}\text { 2-(acetylamino)-1,5- } \\
\text { anhydro-2-deoxy-4-O-b-D- } \\
\text { galactopyranosyl-D-arabino- } \\
\text { Hex-1-enitol }\end{array}$ \\
\hline 14 & 15.590 & $(\mathrm{M}+\mathrm{H})^{+}$ & 171.1087 & 172.1160 & $\mathrm{C}_{9} \mathrm{H}_{17} \mathrm{NS}$ & 90.62 & -3.11 & $\begin{array}{l}\text { 2,5-Dihydro-4,5-dimethyl-2- } \\
\text { (1-methylpropyl)thiazole* }\end{array}$ \\
\hline 15 & 7.631 & $(\mathrm{M}+\mathrm{H})^{+}$ & 291.0958 & 292.1030 & $\mathrm{C}_{11} \mathrm{H}_{17} \mathrm{NO}_{8}$ & 98.24 & -1.31 & $\begin{array}{l}\text { 2,7-Anhydro-alpha-N- } \\
\text { acetylneuraminic acid }\end{array}$ \\
\hline 16 & 8.822 & $\left(\mathrm{M}+\mathrm{NH}_{4}\right)^{+}$ & 418.1835 & 436.2172 & $\mathrm{C}_{19} \mathrm{H}_{30} \mathrm{O}_{10}$ & 98.30 & 1.05 & $\begin{array}{l}\text { 2-[4-(3-Hydroxypropyl)- } \\
\text { 2-methoxyphenoxy]-1,3- } \\
\text { propanediol 1-glucoside }\end{array}$ \\
\hline 17 & 8.685 & $(\mathrm{M}+\mathrm{H})^{+}$ & 113.0843 & 114.0917 & $\mathrm{C}_{6} \mathrm{H}_{11} \mathrm{NO}$ & 86.02 & -2.34 & 2-Acetylpyrrolidine* \\
\hline 18 & 5.701 & $\left(\mathrm{M}+\mathrm{NH}_{4}\right)^{+}$ & 155.0950 & 173.1288 & $\mathrm{C}_{8}^{0} \mathrm{H}_{13} \mathrm{NO}_{2}$ & 86.86 & -2.51 & $\begin{array}{l}\text { 2-Amino-2- } \\
\text { Norbornanecarboxylic acid }\end{array}$ \\
\hline 19 & 5.702 & $(\mathrm{M}+\mathrm{H})^{+}$ & 190.0958 & 191.1029 & $\mathrm{C}_{7} \mathrm{H}_{14} \mathrm{~N}_{2} \mathrm{O}_{4}$ & 90.69 & -2.50 & $\begin{array}{l}\text { 2-Amino-4-[(2-hydroxy-1- } \\
\text { oxopropyl)amino]butanoic } \\
\text { acid }\end{array}$ \\
\hline 20 & 7.725 & $\left(\mathrm{M}+\mathrm{NH}_{4}\right)^{+}$ & 239.1067 & 257.1405 & $\mathrm{C}_{14} \mathrm{H}_{13} \mathrm{~N}_{3} \mathrm{O}$ & 81.23 & -3.70 & $\begin{array}{l}\text { 2-amino-a-phenyl-1H- } \\
\text { Benzimidazole-5-methanol }\end{array}$ \\
\hline 21 & 10.087 & $\left(\mathrm{M}+\mathrm{NH}_{4}\right)^{+}$ & 94.0785 & 112.1124 & $\mathrm{C}_{7} \mathrm{H}_{10}$ & 86.34 & -2.46 & 2-Methyl-1,3-cyclohexadiene \\
\hline 22 & 7.103 & $(\mathrm{M}+\mathrm{H})^{+}$ & 155.0701 & 156.0774 & $\mathrm{C}_{6} \mathrm{H}_{9} \mathrm{~N}_{3} \mathrm{O}_{2}$ & 84.30 & -4.09 & 3-(Pyrazol-1-yl)-L-alanine \\
\hline 23 & 6.949 & $\left(\mathrm{M}+\mathrm{NH}_{4}\right)^{+}$ & 118.0421 & 136.0759 & $\mathrm{C}_{8} \mathrm{H}_{6} \mathrm{O}^{3-2}$ & 87.41 & -1.96 & $3,5,7$-Octatriyn-1-ol \\
\hline 24 & 7.656 & $\left(\mathrm{M}+\mathrm{NH}_{4}\right)^{+}$ & 477.1901 & 495.2239 & $\mathrm{C}_{26}^{8} \mathrm{H}_{27} \mathrm{~N}_{3} \mathrm{O}_{6}$ & 93.78 & -0.31 & $\begin{array}{l}\text { 3,5-Pyridinedicarboxylic } \\
\text { acid, 2,6-dimethyl-4-(3- } \\
\text { nitrophenyl)-, methyl } \\
\text { 2-[methyl(phenylmethyl) } \\
\text { amino] }\end{array}$ \\
\hline 25 & 9.346 & $(\mathrm{M}+\mathrm{H})^{+}$ & 186.1374 & 187.1449 & $\mathrm{C}_{9} \mathrm{H}_{18} \mathrm{~N}_{2} \mathrm{O}_{2}$ & 96.27 & -3.33 & $\begin{array}{l}\text { 3-[(3-Methylbutyl) } \\
\text { nitrosoamino]-2-butanone }\end{array}$ \\
\hline 26 & 6.347 & $\left(\mathrm{M}+\mathrm{NH}_{4}\right)^{+}$ & 129.0791 & 147.1129 & $\mathrm{C}_{6} \mathrm{H}_{11} \mathrm{NO}_{2}$ & 99.35 & -0.98 & 3-acetamidobutanal \\
\hline 27 & 7.395 & $(\mathrm{M}+\mathrm{H})^{+}$ & 194.1061 & 195.1134 & $\mathrm{C}_{10}^{0} \mathrm{H}_{14} \mathrm{~N}_{2} \mathrm{O}_{2}$ & 85.27 & -2.80 & $\begin{array}{l}\text { 3-Hydroxy-N- } \\
\text { glycyl-2,6-xylidine } \\
\text { (3-Hydroxyglycinexylidide) }\end{array}$ \\
\hline 28 & 7.632 & $(\mathrm{M}+\mathrm{H})^{+}$ & 196.0377 & 197.0449 & $\mathrm{C}_{9} \mathrm{H}_{8} \mathrm{O}_{5}$ & 85.10 & -2.44 & $\begin{array}{l}\text { 3-Methoxy- } 4,5- \\
\text { methylenedioxybenzoic acid }\end{array}$ \\
\hline 29 & 19.921 & $\left(\mathrm{M}+\mathrm{NH}_{4}\right)^{+}$ & 278.2972 & 296.3311 & $\mathrm{C}_{20} \mathrm{H}_{38}$ & 85.63 & 0.46 & $3 Z, 6 Z$-Eicosadiene \\
\hline 30 & 10.783 & $(\mathrm{M}+\mathrm{H})^{+}$ & 218.1424 & 219.1495 & $\mathrm{C}_{13}^{20} \mathrm{H}_{18}^{30} \mathrm{~N}_{2} \mathrm{O}$ & 92.62 & & $\begin{array}{l}\text { 4-[2-(Propylamino)ethyl]- } \\
\text { 1,3-dihydro-2H-indol-2-one }\end{array}$ \\
\hline 31 & 7.627 & $(\mathrm{M}+\mathrm{Na})^{+}$ & 145.0766 & 168.0658 & $\mathrm{C}_{9} \mathrm{H}_{9} \mathrm{~N}_{2}$ & 86.63 & 0.02 & 4-Aminomethylindole \\
\hline 32 & 8.253 & $\left(\mathrm{M}+\mathrm{NH}_{4}\right)^{+}$ & 153.1156 & 171.1493 & $\mathrm{C}_{9} \mathrm{H}_{15} \mathrm{NO}$ & 92.51 & -1.46 & 4-Butyl-2,5-dimethyloxazole \\
\hline 33 & 5.641 & $\left(\mathrm{M}+\mathrm{NH}_{4}\right)^{+}$ & 167.1316 & 185.1654 & $\mathrm{C}_{10} \mathrm{H}_{17} \mathrm{NO}$ & 85.56 & -3.51 & $\begin{array}{l}\text { 4-Butyl-2-ethyl-5- } \\
\text { methyloxazole }\end{array}$ \\
\hline 34 & 10.175 & $(\mathrm{M}+\mathrm{H})^{+}$ & 104.0373 & 105.0446 & $\mathrm{C}_{6} \mathrm{H}_{4} \mathrm{~N}_{2}$ & 87.29 & 1.25 & 4-Cyanopyridine \\
\hline 35 & 8.933 & $(\mathrm{M}+\mathrm{H})^{+}$ & 466.2196 & 467.2267 & $\mathrm{C}_{19} \mathrm{H}_{30} \mathrm{D}_{3} \mathrm{~N}_{3} \mathrm{O}_{8} \mathrm{~S}$ & 87.39 & -4.25 & $\begin{array}{l}\text { 4-hydroxy Nonenal } \\
\text { Glutathione-d3 }\end{array}$ \\
\hline 36 & 6.351 & $(\mathrm{M}+\mathrm{H})^{+}$ & 166.0379 & 167.0452 & $\mathrm{C}_{7} \mathrm{H}_{6} \mathrm{~N}_{2} \mathrm{O}_{3}$ & 83.68 & -0.52 & $\begin{array}{l}\text { 4-Hydroxy-3- } \\
\text { nitrosobenzamide }\end{array}$ \\
\hline 37 & 8.457 & $\left(\mathrm{M}+\mathrm{NH}_{4}\right)^{+}$ & 129.0430 & 147.0769 & $\mathrm{C}_{5} \mathrm{H}_{7} \mathrm{NO}_{3}$ & 96.21 & -2.91 & 4-Oxoproline* \\
\hline
\end{tabular}


Table-3: (Continued)

\begin{tabular}{|c|c|c|c|c|c|c|c|c|}
\hline Number & $\underset{(\min )}{\mathbf{R T}}$ & Ion & Mass & $\mathbf{m} / \mathbf{z}$ & $\begin{array}{l}\text { Molecular } \\
\text { formula }\end{array}$ & $\begin{array}{l}\text { Score } \\
\text { (DB) }\end{array}$ & $\begin{array}{l}\text { DB differences } \\
(\mathrm{ppm})\end{array}$ & $\begin{array}{l}\text { Putatively identified } \\
\text { metabolites }\end{array}$ \\
\hline 38 & 6.327 & $\left(\mathrm{M}+\mathrm{NH}_{4}\right)^{+}$ & 168.0903 & 186.1241 & $\mathrm{~N}_{2} \mathrm{O}_{2}$ & 86.78 & -2.26 & 4-PIOL \\
\hline 39 & 8.928 & $\left(\mathrm{M}+\mathrm{NH}_{4}\right)^{+}$ & 139.0638 & 157.0977 & & 86.17 & -3.48 & 5-Acetyl-2,4-dimethyloxazole \\
\hline 40 & 7.722 & $\left(\mathrm{M}+\mathrm{NH}_{4}^{4}\right)^{+}$ & 267.1016 & 285.1354 & ${ }_{3} \mathrm{~N}_{3}^{2} \mathrm{O}_{2}$ & 81.08 & -3.24 & $\begin{array}{l}\text { 5-benzyl-5-(pyridin-3-yl) } \\
\text { imidazolidine-2,4-dione }\end{array}$ \\
\hline 41 & 7.815 & $\left(\mathrm{M}+\mathrm{NH}_{4}\right)^{+}$ & 165.1159 & 183.1497 & $\mathrm{C}_{10} \mathrm{H}_{15} \mathrm{NO}$ & 98.24 & -3.05 & $\begin{array}{l}\text { 5-Methyl-2-(1-pyrrolidinyl)- } \\
\text { 2-cyclopenten-1-one* }\end{array}$ \\
\hline 42 & 7.400 & $(\mathrm{M}+\mathrm{H})^{+}$ & 241.1067 & 242.1140 & $\mathrm{C}_{10} \mathrm{H}_{15} \mathrm{~N}_{3} \mathrm{O}_{4}$ & 86.34 & -1.88 & 5-Methyldeoxycytidine \\
\hline 43 & 9.361 & $(\mathrm{M}+\mathrm{Na})^{+}$ & 597.3489 & 620.3383 & $\mathrm{C}_{31} \mathrm{H}_{51} \mathrm{NO}_{10}$ & 89.94 & 4.09 & $\begin{array}{l}\text { 5-O- } \beta \text {-D- } \\
\text { Mycaminosyltylonolide }\end{array}$ \\
\hline 44 & 7.658 & $\left(\mathrm{M}+\mathrm{NH}_{4}\right)^{+}$ & 139.0999 & 157.1337 & $\mathrm{C}_{8} \mathrm{H}_{13} \mathrm{NO}$ & 92.23 & -1.66 & 5-Pentyloxazole \\
\hline 45 & 7.406 & $(\mathrm{M}+\mathrm{H})^{+}$ & 172.0851 & 173.0924 & ${ }_{2} \mathrm{~N}_{2} \mathrm{O}_{3}$ & 97.82 & -1.94 & 5- $\delta$-Hydroxybutyl Hydantoin \\
\hline 46 & 8.150 & $(\mathrm{M}+\mathrm{Na})^{+}$ & 585.2945 & 608.2836 & ${ }_{39} \mathrm{~N}_{5} \mathrm{O}_{5}$ & 91.39 & 1.04 & $\begin{array}{l}8,10^{\prime}- \\
\text { Dihydroxydihydroergotamine }\end{array}$ \\
\hline 47 & 6.927 & $(\mathrm{M}+\mathrm{H})^{+}$ & 216.0755 & 217.0829 & $\mathrm{~V}_{2} \mathrm{O}_{5}$ & 95.52 & -4.14 & 8-Hydroxyalanylclavam \\
\hline 48 & 7.919 & $\left(\mathrm{M}+\mathrm{NH}_{4}\right)^{+}$ & 228.1364 & 246.1703 & & 86.63 & -1.26 & 9,12-dioxo-dodecanoic acid \\
\hline 49 & 16.039 & $\left(\mathrm{M}+\mathrm{NH}_{4}^{4}\right)^{+}$ & 250.2300 & 268.2639 & & 86.18 & -1.19 & $\begin{array}{l}\text { 9S,10R-Epoxy-3Z, } \\
\text { 6Z-octadecadiene* }\end{array}$ \\
\hline 50 & 19.926 & $\left(\mathrm{M}+\mathrm{NH}_{4}\right)^{+}$ & 364.4068 & 382.4407 & & 99.78 & 0.22 & 9Z-Hexacosene* \\
\hline 51 & 6.756 & $\left(\mathrm{M}+\mathrm{NH}_{4}^{4}\right)^{+}$ & 217.0856 & 235.1194 & $\mathrm{~N}_{3} \mathrm{O}_{2}$ & 86.25 & -2.08 & Acetylhydrazinopthalazinone \\
\hline 52 & 9.279 & $\left(\mathrm{M}+\mathrm{NH}_{4}\right)^{+}$ & 245.1170 & 263.1508 & ${ }_{5} \mathrm{~N}_{3} \mathrm{O}_{2}^{2}$ & 96.87 & -2.15 & Acetyltryptophanamide \\
\hline 53 & 4.538 & $(\mathrm{M}+\mathrm{H})^{+}$ & 135.0551 & 136.0623 & $\mathrm{C}_{5}$ & 84.51 & -4.22 & Adenine* \\
\hline 54 & 7.198 & $(\mathrm{M}+\mathrm{H})^{+}$ & 243.1221 & 244.1298 & $\mathrm{~N}_{3} \mathrm{O}_{4}$ & 85.40 & -0.95 & Ala Gly Pro \\
\hline 55 & 6.934 & $\left(\mathrm{M}+\mathrm{NH}_{4}\right)^{+}$ & 236.1165 & 254.1504 & $\mathrm{~N}_{2} \mathrm{O}_{3}$ & 97.31 & -1.88 & Alanyl-DL-Phenylalanine \\
\hline 56 & 3.734 & $(\mathrm{M}+\mathrm{H})^{+}$ & 226.1074 & 227.1145 & $\mathrm{~N}_{4} \mathrm{O}_{3}$ & 87.75 & -3.48 & Alanyl-Histidine* \\
\hline 57 & 8.374 & $(\mathrm{M}+\mathrm{H})^{+}$ & 232.1578 & 233.1652 & $\mathrm{~N}_{2} \mathrm{O}$ & 85.03 & -0.85 & Albine \\
\hline 58 & 6.159 & $\left(\mathrm{M}+\mathrm{NH}_{4}\right)^{+}$ & 141.0796 & 159.1134 & $\mathrm{NO}_{2}$ & 85.48 & -4.19 & Arecaidine \\
\hline 59 & 7.686 & $(\mathrm{M}+\mathrm{H})^{+}$ & 271.1650 & 272.1723 & ${ }_{1} \mathrm{~N}_{5}^{2} \mathrm{O}_{3}$ & 86.06 & -1.91 & Arginyl-Proline \\
\hline 60 & 7.155 & $(\mathrm{M}+\mathrm{H})^{+}$ & 279.1216 & 280.1289 & $\mathrm{~N}_{3} \mathrm{O}_{4}^{3}$ & 85.73 & 1.14 & Asn Phe \\
\hline 61 & 9.361 & $\left(\mathrm{M}+\mathrm{NH}_{4}\right)^{+}$ & 378.1906 & 396.2245 & $\mathrm{~N}_{4} \mathrm{O}_{5}$ & 99.55 & -0.62 & Asn Val Phe \\
\hline 62 & 5.759 & $(\mathrm{M}+\mathrm{H})^{+}$ & 368.1330 & 369.1404 & ${ }_{0} \mathrm{~N}_{4} \mathrm{O}_{7}^{5}$ & 84.38 & 0.41 & Asn-Lys-OH \\
\hline 63 & 3.515 & $(\mathrm{M}+\mathrm{Na})^{+}$ & 349.1122 & 372.1015 & ${ }_{9} \mathrm{~N}_{3} \mathrm{O}_{9}$ & 98.31 & -0.19 & Asp Thr Asp \\
\hline 64 & 7.189 & $(\mathrm{M}+\mathrm{Na})^{+}$ & 559.3144 & 582.3037 & ${ }_{5} \mathrm{NO}_{8}$ & 94.22 & 0.15 & Auriculine \\
\hline 65 & 3.073 & $\left(\mathrm{M}+\mathrm{NH}_{4}\right)^{+}$ & 173.0434 & 191.0772 & $\mathrm{~V}_{3} \mathrm{O}_{4}^{8}$ & 87.00 & 1.51 & Azaserine \\
\hline 66 & 14.410 & $\left(\mathrm{M}+\mathrm{NH}_{4}^{4}\right)^{+}$ & 290.1788 & 308.2126 & & 83.40 & -1.75 & Azatadine \\
\hline 67 & 4.411 & $\left(\mathrm{M}+\mathrm{NH}_{4}^{4}\right)^{+}$ & 472.2283 & 490.2622 & ${ }_{5}^{2} \mathrm{O}_{5}^{2} \mathrm{~S}$ & 93.08 & 0.12 & BAY-u9773 \\
\hline 68 & 7.655 & $\left(\mathrm{M}+\mathrm{NH}_{4}\right)^{+}$ & 165.0796 & 183.1135 & $\mathrm{NO}_{2}$ & 85.21 & -4.05 & Benzocaine \\
\hline 69 & 9.276 & $(\mathrm{M}+\mathrm{H})^{+}$ & 234.1483 & 235.1557 & ${ }_{18} \mathrm{~N}_{4} \mathrm{O}$ & 94.24 & -1.09 & Benzoylagmatine \\
\hline 70 & 8.382 & $(\mathrm{M}+\mathrm{Na})^{+}$ & 365.1733 & 388.1628 & $\mathrm{C}_{21} \mathrm{H}_{23} \mathrm{~N}_{3} \mathrm{O}_{3}$ & 82.56 & 1.83 & Brevianamide B \\
\hline 71 & 3.342 & $(\mathrm{M}+\mathrm{H})^{+}$ & 109.0644 & 110.0717 & & 96.49 & -4.13 & Brunfelsamidine* \\
\hline 72 & 8.118 & $(\mathrm{M}+\mathrm{H})^{+}$ & 643.3329 & 644.3407 & $\mathrm{C}_{35} \mathrm{H}_{49} \mathrm{NO}_{10}$ & 80.90 & 4.29 & $\begin{array}{l}\text { Buprenorphine } \\
\text { 3-O-glucuronide }\end{array}$ \\
\hline 73 & 7.511 & $\left(\mathrm{M}+\mathrm{NH}_{4}\right)^{+}$ & 224.1165 & 242.1504 & ${ }_{6} \mathrm{~N}_{2} \mathrm{O}_{3}$ & 84.08 & -2.03 & Butalbital \\
\hline 74 & 6.960 & $(\mathrm{M}+\mathrm{H})^{+}$ & 212.1166 & 213.1240 & ${ }_{16}^{16} \mathrm{~N}_{2} \mathrm{O}_{3}$ & 80.31 & -2.26 & Butethal \\
\hline 75 & 6.757 & $(\mathrm{M}+\mathrm{H})^{+}$ & 278.1270 & 279.1342 & $\mathrm{C}_{14} \mathrm{H}_{18} \mathrm{~N}_{2} \mathrm{O}_{4}$ & 95.45 & -1.14 & Carboxy-PTIO \\
\hline 76 & 8.511 & $(\mathrm{M}+\mathrm{H})^{+}$ & 607.3124 & 608.3200 & $\mathrm{C}_{31}^{14} \mathrm{H}_{41} \mathrm{~N}_{7} \mathrm{O}_{6}$ & 90.53 & -0.90 & Chymostatin \\
\hline 77 & 17.086 & $(\mathrm{M}+\mathrm{H})^{+}$ & 127.1365 & 128.1437 & & 86.60 & -2.89 & Coniine* \\
\hline 78 & 6.940 & $(\mathrm{M}+\mathrm{H})^{+}$ & 176.0947 & 177.1028 & $\mathrm{C}_{10}^{8} \mathrm{H}_{12} \mathrm{~N}_{2} \mathrm{O}$ & 82.06 & 1.45 & Cotinine \\
\hline 79 & 9.360 & $(\mathrm{M}+\mathrm{Na})^{+}$ & 203.1303 & 226.1195 & $\mathrm{C}_{13} \mathrm{H}_{17} \mathrm{NO}$ & 96.10 & 3.31 & Crotamiton \\
\hline 80 & 9.356 & $(\mathrm{M}+\mathrm{H})^{+}$ & 224.1892 & 225.1965 & ${ }_{4} \mathrm{~N}_{2} \mathrm{O}$ & 93.91 & -1.45 & Cuscohygrine \\
\hline 81 & 7.537 & $(\mathrm{M}+\mathrm{H})^{+}$ & 244.1215 & 245.1287 & $\mathrm{C}_{14}^{13} \mathrm{H}_{16} \mathrm{~N}_{2} \mathrm{O}_{2}$ & 86.62 & -1.11 & Cyclo(L-Phe-L-Pro) \\
\hline 82 & 6.992 & $(\mathrm{M}+\mathrm{H})^{+}$ & 227.0914 & 228.0986 & $\mathrm{C}_{9} \mathrm{H}_{13} \mathrm{~N}_{3} \mathrm{O}_{4}^{2}$ & 95.88 & -3.67 & Deoxycytidine \\
\hline 83 & 9.360 & $(\mathrm{M}+\mathrm{H})^{+}$ & 249.1484 & 250.1556 & $\mathrm{C}_{13} \mathrm{H}_{19} \mathrm{~N}_{3} \mathrm{O}_{2}$ & 93.27 & -2.74 & $\begin{array}{l}\text { Desethyl-N } \\
\text {-acetylprocainamide }\end{array}$ \\
\hline 84 & 8.511 & $(\mathrm{M}+\mathrm{H})^{+}$ & 308.1534 & 309.1601 & $\mathrm{~N}_{2} \mathrm{O}_{2}$ & 86.91 & -3.03 & $D M X B-A$ \\
\hline 85 & 5.307 & $(\mathrm{M}+\mathrm{H})^{+}$ & 115.0636 & 116.0709 & $\mathrm{jO}_{2}$ & 92.37 & -2.15 & D-Proline \\
\hline 86 & 7.816 & $(\mathrm{M}+\mathrm{H})^{+}$ & 491.2724 & 492.2797 & $\mathrm{C}_{27} \mathrm{H}_{41} \mathrm{NO}_{5} \mathrm{~S}$ & 86.49 & -3.72 & Epothilone D \\
\hline 87 & 6.928 & $(\mathrm{M}+\mathrm{H})^{+}$ & 204.0900 & 205.0974 & ${ }_{2}^{4} \mathrm{~N}_{2} \mathrm{O}_{2}$ & 83.85 & -0.71 & Ethotoin \\
\hline 88 & 8.746 & $\left(\mathrm{M}+\mathrm{NH}_{4}\right)^{+}$ & 261.1004 & 279.1341 & $\mathrm{C}_{14}^{11} \mathrm{H}_{15} \mathrm{NO}_{4}^{2}$ & 85.03 & -0.98 & $\begin{array}{l}\text { Ethyl 1-benzyl-3- } \\
\text { hydroxy- 2-oxo[5H] } \\
\text { pyrrole-4-carboxylate }\end{array}$ \\
\hline 89 & 9.297 & $\left(\mathrm{M}+\mathrm{NH}_{4}\right)^{+}$ & 373.1958 & 391.2294 & ${ }_{27} \mathrm{~N}_{5} \mathrm{O}_{6}$ & 83.01 & 0.87 & GIn Asn Ile \\
\hline 90 & 6.351 & $(\mathrm{M}+\mathrm{H})^{+}$ & 300.1433 & 301.1505 & $\mathrm{C}_{12} \mathrm{H}_{20} \mathrm{~N}_{4} \mathrm{O}_{5}$ & 99.12 & 0.11 & GIn Gly Pro \\
\hline 91 & 7.242 & $(\mathrm{M}+\mathrm{H})^{+}$ & 406.2211 & 407.2284 & $\mathrm{C}_{20} \mathrm{H}_{30} \mathrm{~N}_{4} \mathrm{O}_{5}^{5}$ & 81.92 & 1.31 & GIn Leu Phe \\
\hline 92 & 9.361 & $(\mathrm{M}+\mathrm{H})^{+}$ & 421.2328 & 422.2402 & $\mathrm{~N}_{5} \mathrm{O}_{5}$ & 96.22 & -0.75 & GIn Phe Lys \\
\hline 93 & 7.815 & $(\mathrm{M}+\mathrm{H})^{+}$ & 356.2061 & 357.2133 & $\mathrm{C}_{16} \mathrm{H}_{28}^{31} \mathrm{~N}_{4}^{5} \mathrm{O}_{5}^{5}$ & 98.89 & -0.50 & GIn Pro Leu \\
\hline 94 & 7.150 & $(\mathrm{M}+\mathrm{Na})^{+}$ & 340.1749 & 363.1642 & $\mathrm{C}_{15} \mathrm{H}_{24} \mathrm{~N}_{4} \mathrm{O}_{5}$ & 84.85 & -0.70 & GIn Pro Pro \\
\hline
\end{tabular}


Table-3: (Continued)

\begin{tabular}{|c|c|c|c|c|c|c|c|c|}
\hline Number & $\begin{array}{l}\text { RT } \\
(\min )\end{array}$ & Ion & Mass & $\mathbf{m} / \mathbf{z}$ & $\begin{array}{l}\text { Molecular } \\
\text { formula }\end{array}$ & $\begin{array}{l}\text { Score } \\
\text { (DB) }\end{array}$ & $\begin{array}{c}\text { DB differences } \\
(\mathrm{ppm})\end{array}$ & $\begin{array}{l}\text { Putatively identified } \\
\text { metabolites }\end{array}$ \\
\hline$\overline{95}$ & 6.983 & $\left(\mathrm{M}+\mathrm{NH}_{4}\right)^{+}$ & 462.1757 & 480.2096 & $\mathrm{C}_{21} \mathrm{H}_{26} \mathrm{~N}_{4} \mathrm{O}_{8}$ & 81.05 & -1.42 & Glu Trp Glu \\
\hline 96 & 7.169 & $(\mathrm{M}+\mathrm{H})^{+}$ & 172.0854 & 173.0927 & $\mathrm{~N}_{2} \mathrm{O}_{3}$ & 97.76 & -3.55 & Gly Pro \\
\hline 97 & 8.482 & $\left(\mathrm{M}+\mathrm{NH}_{4}\right)^{+}$ & 311.1121 & 329.1458 & $\mathrm{C}_{13} \mathrm{H}_{17} \mathrm{~N}_{3} \mathrm{O}_{6}$ & 82.80 & -1.26 & Gly-Lys-OH \\
\hline 98 & 14.553 & $(\mathrm{M}+\mathrm{H})^{+}$ & 273.2672 & 274.2746 & $\mathrm{C}_{16} \mathrm{H}_{35} \mathrm{NO}_{2}$ & 95.51 & -1.71 & Hexadecasphinganine \\
\hline 99 & 18.610 & $(\mathrm{M}+\mathrm{H})^{+}$ & 101.1200 & 102.1273 & & 84.30 & 3.97 & Hexylamine* \\
\hline 100 & 5.759 & $(\mathrm{M}+\mathrm{H})^{+}$ & 226.1065 & 227.1137 & $\mathrm{C}_{9} \mathrm{H}_{14} \mathrm{~N}_{4} \mathrm{O}_{3}$ & 99.39 & 0.42 & His Ala \\
\hline 101 & 8.072 & $(\mathrm{M}+\mathrm{H})^{+}$ & 302.1386 & 303.1461 & $\mathrm{C}_{15} \mathrm{H}_{18} \mathrm{~N}_{4} \mathrm{O}_{3}$ & 82.69 & -2.51 & His Phe \\
\hline 102 & 7.651 & $(\mathrm{M}+\mathrm{H})^{+}$ & 417.2011 & 418.2082 & ${ }_{27}^{18} \mathrm{~N}_{5}^{4} \mathrm{O}_{5}^{3}$ & 96.58 & 0.17 & His Tyr Val \\
\hline 103 & 7.209 & $\left(\mathrm{M}+\mathrm{NH}_{4}\right)^{+}$ & 348.1060 & 366.1397 & ${ }_{6}^{7} \mathrm{~N}_{4}^{5} \mathrm{O}_{6}^{5}$ & 94.60 & 2.81 & His-Ala-OH \\
\hline 104 & 8.180 & $(\mathrm{M}+\mathrm{H})^{+}$ & 302.1382 & 303.1453 & $\mathrm{C}_{15}^{15} \mathrm{H}_{18} \mathrm{~N}_{4} \mathrm{O}_{3}^{6}$ & 84.08 & -1.15 & Histidinyl-Phenylalanine \\
\hline 105 & 5.675 & $\left(\mathrm{M}+\mathrm{NH}_{4}\right)^{+}$ & 254.1383 & 272.1722 & $\mathrm{C}_{11}^{15} \mathrm{H}_{18} \mathrm{~N}_{4} \mathrm{O}_{3}$ & 98.61 & -1.64 & Histidinyl-Valine \\
\hline 106 & 6.641 & $(\mathrm{M}+\mathrm{H})^{+}$ & 259.1168 & 260.1239 & $\mathrm{C}_{10} \mathrm{H}_{17} \mathrm{~N}_{3} \mathrm{O}_{5}$ & 83.20 & 0.08 & $\begin{array}{l}\text { Hydroxypropyl-Gamma- } \\
\text { glutamate }\end{array}$ \\
\hline 107 & 7.566 & $\left(\mathrm{M}+\mathrm{NH}_{4}\right)^{+}$ & 268.1173 & 286.1510 & $\mathrm{C}_{11} \mathrm{H}_{16} \mathrm{~N}_{4} \mathrm{O}_{4}$ & 81.20 & -0.36 & Hydroxypropyl-Histidine \\
\hline 108 & 5.700 & $(\mathrm{M}+\mathrm{Na})^{+}$ & 289.1639 & 312.1534 & $\mathrm{C}_{12} \mathrm{H}_{23} \mathrm{~N}_{3} \mathrm{O}_{5}^{4}$ & 81.82 & -0.36 & Ile Ala Ser \\
\hline 109 & 7.815 & $(\mathrm{M}+\mathrm{H})^{+}$ & 259.1538 & 260.1611 & $\mathrm{C}_{11}^{12} \mathrm{H}_{21} \mathrm{~N}_{3} \mathrm{O}_{4}^{5}$ & 98.35 & -2.20 & Ile Gln \\
\hline 110 & 8.447 & $(\mathrm{M}+\mathrm{H})^{+}$ & 356.2061 & 357.2134 & $\mathrm{C}_{16}^{11} \mathrm{H}_{28} \mathrm{~N}_{4} \mathrm{O}_{5}^{4}$ & 94.09 & -0.48 & Ile Gln Pro \\
\hline 111 & 6.481 & $\left(\mathrm{M}+\mathrm{NH}_{4}\right)^{+}$ & 180.0538 & 198.0876 & $\mathrm{C}_{8} \mathrm{H}_{8} \mathrm{~N}_{2} \mathrm{O}_{3}$ & 85.24 & -1.88 & Isonicotinylglycine \\
\hline 112 & 18.618 & $(\mathrm{M}+\mathrm{H})^{+}$ & 298.1545 & 299.1616 & $\mathrm{C}_{15}^{0} \mathrm{H}_{18} \mathrm{~N}_{6} \mathrm{O}$ & 87.32 & -0.95 & Iso-Olomoucine* \\
\hline 113 & 8.688 & $\left(\mathrm{M}+\mathrm{NH}_{4}\right)^{+}$ & 268.1315 & 286.1653 & $\mathrm{C}_{14} \mathrm{H}_{20} \mathrm{O}_{5}$ & 95.31 & -1.49 & Kamahine C* \\
\hline 114 & 3.088 & $\left(\mathrm{M}+\mathrm{NH}_{4}\right)^{+}$ & 151.0606 & 169.0945 & $\mathrm{C}_{5} \mathrm{H}_{11} \mathrm{O}_{5}$ & 83.97 & 0.54 & $\mathrm{~L}-(+)$-Arabinose \\
\hline 115 & 9.360 & $(\mathrm{M}+\mathrm{H})^{+}$ & 210.1373 & 211.1445 & $\mathrm{C}_{11} \mathrm{H}_{18} \mathrm{~N}_{2} \mathrm{O}_{2}$ & 97.17 & -2.43 & L,L-Cyclo(leucylprolyl) \\
\hline 116 & 3.379 & $(\mathrm{M}+\mathrm{Na})^{+}$ & 383.1427 & 406.1318 & $\mathrm{C}_{14}^{11} \mathrm{H}_{25} \mathrm{NO}_{11}$ & 82.74 & 0.16 & Lacto-N-biose I* \\
\hline 117 & 6.715 & $(\mathrm{M}+\mathrm{H})^{+}$ & 196.1218 & 197.1292 & $\mathrm{C}_{10}^{14} \mathrm{H}_{16}^{25} \mathrm{~N}_{2} \mathrm{O}_{2}$ & 81.16 & -3.27 & $\begin{array}{l}\text { L-alpha-Amino- } 1 \mathrm{H} \\
\text {-pyrrole-1-hexanoic acid }\end{array}$ \\
\hline 118 & 9.355 & $(\mathrm{M}+\mathrm{H})^{+}$ & 372.2377 & 373.2447 & ${ }_{2} \mathrm{~N}_{4} \mathrm{O}_{5}$ & 84.53 & -1.09 & Leu Ile Gln \\
\hline 119 & 16.538 & $(\mathrm{M}+\mathrm{H})^{+}$ & 195.0538 & 196.0610 & $\mathrm{C}_{9} \mathrm{H}_{9} \mathrm{NO}_{4}$ & 83.37 & -3.51 & Leucodopachrome \\
\hline 120 & 6.993 & $(\mathrm{M}+\mathrm{H})^{+}$ & 259.1905 & 260.1976 & $\mathrm{C}_{12}^{9} \mathrm{H}_{25} \mathrm{~N}_{3}^{4} \mathrm{O}_{3}$ & 80.87 & -3.52 & Leucyl-Lysine \\
\hline 121 & 8.258 & $(\mathrm{M}+\mathrm{H})^{+}$ & 280.1065 & 281.1142 & $\mathrm{C}_{14}^{12} \mathrm{H}_{12} \mathrm{~N}_{6} \mathrm{O}^{3}$ & 81.98 & 2.63 & Levosimendan \\
\hline 122 & 8.072 & $(\mathrm{M}+\mathrm{H})^{+}$ & 587.3072 & 588.3144 & $\mathrm{C}_{32}{ }^{14} \mathrm{H}_{45} \mathrm{NO}_{9}$ & 84.74 & 3.76 & Lipomycin \\
\hline 123 & 9.360 & $(\mathrm{M}+\mathrm{H})^{+}$ & 252.1846 & 253.1918 & $\mathrm{C}_{14}^{32} \mathrm{H}_{24}^{45} \mathrm{~N}_{2} \mathrm{O}_{2}$ & 96.56 & -3.25 & Lupanyl Acid \\
\hline 124 & 7.815 & $\left(\mathrm{M}+\mathrm{NH}_{4}\right)^{+}$ & 396.2010 & 414.2348 & $\mathrm{C}_{18}^{14} \mathrm{H}_{28}^{24} \mathrm{~N}_{4}^{2} \mathrm{O}_{6}^{2}$ & 99.69 & -0.28 & Lys Ser Tyr \\
\hline 125 & 6.826 & $(\mathrm{M}+\mathrm{H})^{+}$ & 309.1685 & 310.1762 & $\mathrm{C}_{15}^{18} \mathrm{H}_{23}^{28} \mathrm{~N}_{3} \mathrm{O}_{4}^{6}$ & 87.90 & 1.22 & Lys Tyr \\
\hline 126 & 4.413 & $(\mathrm{M}+\mathrm{H})^{+}$ & 233.1375 & 234.1451 & $\mathrm{C}_{9} \mathrm{H}_{19} \mathrm{~N}_{3} \mathrm{O}_{4}$ & 93.49 & 0.10 & Lysinoalanine \\
\hline 127 & 7.814 & $\left(\mathrm{M}+\mathrm{NH}_{4}\right)^{+}$ & 293.1746 & 311.2084 & $\mathrm{C}_{15} \mathrm{H}_{23} \mathrm{~N}_{3} \mathrm{O}_{3}$ & 98.62 & -2.09 & Lysyl-Phenylalanine \\
\hline 128 & 7.162 & $(\mathrm{M}+\mathrm{H})^{+}$ & 309.1693 & 310.1766 & $\mathrm{C}_{15}^{15} \mathrm{H}_{23} \mathrm{~N}_{3} \mathrm{O}_{4}^{3}$ & 98.16 & -1.39 & Lysyl-Tyrosine \\
\hline 129 & 8.464 & $\left(\mathrm{M}+\mathrm{NH}_{4}\right)^{+}$ & 109.0530 & 127.0869 & $\mathrm{C}_{6} \mathrm{H}_{7} \mathrm{NO}^{23}$ & 84.94 & -2.26 & m-Aminophenol \\
\hline 130 & 3.516 & $(\mathrm{M}+\mathrm{H})^{+}$ & 114.0432 & 115.0504 & $\mathrm{C}_{4} \mathrm{H}_{6} \mathrm{~N}_{2} \mathrm{O}_{2}$ & 84.07 & -2.51 & Muscimol \\
\hline 131 & 8.440 & $(\mathrm{M}+\mathrm{H})^{+}$ & 517.2874 & 518.2948 & $\mathrm{C}_{25}^{4} \mathrm{H}_{43} \mathrm{NO}_{10}^{2}$ & 96.46 & 2.44 & Mycalamide B \\
\hline 132 & 7.630 & $(\mathrm{M}+\mathrm{H})^{+}$ & 203.0800 & 204.0871 & $\mathrm{C}_{8} \mathrm{H}_{13}{ }^{3} \mathrm{NO}_{5}$ & 95.68 & -2.92 & N2-Acetyl-L-aminoadipate \\
\hline 133 & 7.512 & $(\mathrm{M}+\mathrm{H})^{+}$ & 130.1109 & 131.1183 & $\mathrm{C}_{6} \mathrm{H}_{14} \mathrm{~N}_{2} \mathrm{O}$ & 86.27 & -2.52 & $\mathrm{~N}$-Acetylputrescine \\
\hline 134 & 6.990 & $(\mathrm{M}+\mathrm{Na})^{+}$ & 175.0989 & 198.0882 & $\mathrm{C}_{11} \mathrm{H}_{13} \mathrm{NO}$ & 82.86 & 4.87 & $\mathrm{~N}$-Acetyltranylcypromine \\
\hline 135 & 7.539 & $(\mathrm{M}+\mathrm{H})^{+}$ & 216.1268 & 217.1341 & $\mathrm{C}_{13}^{11} \mathrm{H}_{16}^{13} \mathrm{~N}_{2} \mathrm{O}$ & 85.32 & -2.70 & $\begin{array}{l}\mathrm{Nb}-\text { Acetyl-Nb- } \\
\text { methyltryptamine }\end{array}$ \\
\hline 136 & 8.747 & $(\mathrm{M}+\mathrm{H})^{+}$ & 135.0686 & 136.0759 & $\mathrm{C} 8 \mathrm{H} 9 \mathrm{NO}$ & 87.70 & -1.39 & N-Benzylformamide \\
\hline 137 & 9.361 & $\left(\mathrm{M}+\mathrm{NH}_{4}\right)^{+}$ & 242.1275 & 260.1613 & $\mathrm{C}_{11} \mathrm{H}_{18} \mathrm{~N}_{2} \mathrm{O}_{4}$ & 97.30 & -3.29 & N-Hydroxypentobarbital \\
\hline 138 & 19.799 & $(\mathrm{M}+\mathrm{Na})^{+}$ & 484.3385 & 507.3275 & $\mathrm{C}_{23} \mathrm{H}_{44}^{10} \mathrm{~N}_{6} \mathrm{O}_{5}^{4}$ & 96.28 & -2.34 & $\begin{array}{l}\text { N-tert-Butyloxycarbonyl- } \\
\text { deacetyl-leupeptin }\end{array}$ \\
\hline 139 & 19.253 & $(\mathrm{M}+\mathrm{H})^{+}$ & 129.1519 & 130.1592 & $\mathrm{C}_{8} \mathrm{r}$ & 99.07 & -1.35 & Octylamine* \\
\hline 140 & 15.006 & $(\mathrm{M}+\mathrm{H})^{+}$ & 255.2568 & 256.2641 & $\mathrm{C}_{16} \mathrm{H}_{33} \mathrm{NO}$ & 95.59 & -2.40 & Palmitic amide* \\
\hline 141 & 9.281 & $(\mathrm{M}+\mathrm{H})^{+}$ & 135.0795 & 136.0868 & $\mathrm{C}_{7} \mathrm{H}_{9} \mathrm{~N}_{3}$ & 94.18 & 0.93 & p-Aminobenzamidine \\
\hline 142 & 10.483 & $(\mathrm{M}+\mathrm{H})^{+}$ & 434.2643 & 435.2716 & $\mathrm{C}_{21} \mathrm{H}_{34} \mathrm{~N}_{6} \mathrm{O}_{4}$ & 92.06 & -0.34 & Phe Arg Leu \\
\hline 143 & 8.862 & $\left(\mathrm{M}+\mathrm{NH}_{4}\right)^{+}$ & 321.1695 & 339.2035 & $\mathrm{C}_{16}^{21} \mathrm{H}_{23} \mathrm{~N}_{3} \mathrm{O}_{4}$ & 92.71 & -2.04 & Phe Gly Val \\
\hline 144 & 9.361 & $\left(\mathrm{M}+\mathrm{NH}_{4}\right)^{+}$ & 406.2583 & 424.2921 & $\mathrm{C}_{21}^{16} \mathrm{H}_{34}^{23} \mathrm{~N}_{4}^{3} \mathrm{O}_{4}^{4}$ & 99.62 & -0.75 & Phe Lys Leu \\
\hline 145 & 12.812 & $(\mathrm{M}+\mathrm{H})^{+}$ & 243.1991 & 244.2065 & $\mathrm{C}_{17}^{21} \mathrm{H}_{25}^{34} \mathrm{~N}^{4-4}$ & 85.35 & -1.83 & Phencyclidine \\
\hline 146 & 18.627 & $(\mathrm{M}+\mathrm{H})^{+}$ & 123.9925 & 124.9998 & $\mathrm{C}_{2} \mathrm{H}_{5} \mathrm{O}_{4} \mathrm{P}$ & 99.77 & 0.25 & Phosphonoacetaldehyde \\
\hline 147 & 7.154 & $(\mathrm{M}+\mathrm{Na})^{+}$ & 542.2482 & 565.2379 & $\mathrm{C}_{23}^{2} \mathrm{H}_{43}^{5} \mathrm{O}_{12} \mathrm{P}$ & 83.84 & 1.95 & $\mathrm{PI}(14: 1(9 \mathrm{Z}) / 0: 0)$ \\
\hline 148 & 8.073 & $(\mathrm{M}+\mathrm{H})^{+}$ & 245.1633 & 246.1708 & $\mathrm{C}_{13}^{23} \mathrm{H}_{19}^{43} \mathrm{~N}_{5}$ & 80.56 & 3.12 & Pinacidil \\
\hline 149 & 7.633 & $\left(\mathrm{M}+\mathrm{NH}_{4}\right)^{+}$ & 256.0588 & 274.0926 & $\mathrm{C}_{11}^{13} \mathrm{H}_{12} \mathrm{O}_{7}^{3}$ & 98.80 & -2.01 & Piscidic Acid \\
\hline 150 & 9.364 & $(\mathrm{M}+\mathrm{Na})^{+}$ & 162.1400 & 185.1293 & $\mathrm{C}_{12}^{11} \mathrm{H}_{18}^{12}$ & 84.10 & 4.97 & Pregeijerene \\
\hline 151 & 7.517 & $(\mathrm{M}+\mathrm{H})^{+}$ & 186.1008 & 187.1084 & $\mathrm{C}_{8} \mathrm{H}_{14} \mathrm{~N}_{2} \mathrm{O}_{3}$ & 84.73 & -1.94 & Pro Ala \\
\hline 152 & 6.991 & $(\mathrm{M}+\mathrm{H})^{+}$ & 326.1598 & 327.1669 & $\mathrm{C}_{14}^{8} \mathrm{H}_{22} \mathrm{~N}_{4} \mathrm{O}_{5}$ & 90.54 & -2.52 & Pro Asn Pro \\
\hline 153 & 6.934 & $(\mathrm{M}+\mathrm{H})^{+}$ & 371.2166 & 372.2239 & $\mathrm{C}_{16}^{14} \mathrm{H}_{29}^{22} \mathrm{~N}_{5}^{4} \mathrm{O}_{5}^{5}$ & 83.53 & 0.79 & Pro GIn Lys \\
\hline 154 & 7.164 & $(\mathrm{M}+\mathrm{H})^{+}$ & 340.1752 & 341.1822 & $\mathrm{C}_{15}^{16} \mathrm{H}_{24} \mathrm{~N}_{4} \mathrm{O}_{5}$ & 92.07 & -1.60 & Pro GIn Pro \\
\hline 155 & 7.402 & $(\mathrm{M}+\mathrm{H})^{+}$ & 269.1378 & 270.1451 & $\mathrm{C}_{12}^{15} \mathrm{H}_{19}^{24} \mathrm{~N}_{3}^{4} \mathrm{O}_{4}^{5}$ & 99.48 & -1.05 & Pro Gly Pro \\
\hline 156 & 7.129 & $(\mathrm{M}+\mathrm{H})^{+}$ & 212.1165 & 213.1239 & $\mathrm{C}_{10}^{12} \mathrm{H}_{16} \mathrm{~N}_{2} \mathrm{O}_{3}$ & 83.55 & -2.05 & Pro Pro \\
\hline
\end{tabular}


Table-3: (Continued)

\begin{tabular}{|c|c|c|c|c|c|c|c|c|}
\hline Number & $\underset{(\min )}{\mathbf{R T}}$ & Ion & Mass & $\mathbf{m} / \mathbf{z}$ & $\begin{array}{l}\text { Molecular } \\
\text { formula }\end{array}$ & $\begin{array}{l}\text { Score } \\
\text { (DB) }\end{array}$ & $\begin{array}{l}\text { DB differences } \\
(\mathrm{ppm})\end{array}$ & $\begin{array}{l}\text { Putatively identified } \\
\text { metabolites }\end{array}$ \\
\hline 157 & 6.930 & $(\mathrm{M}+\mathrm{H})^{+}$ & 269.1372 & 270.1446 & $\mathrm{C}_{12} \mathrm{H}_{19} \mathrm{~N}_{3} \mathrm{O}_{4}$ & 84.07 & 1.16 & Pro Pro Gly \\
\hline 158 & 8.222 & $(\mathrm{M}+\mathrm{H})^{+}$ & 375.1797 & 376.1867 & $\mathrm{C}_{19}^{12} \mathrm{H}_{25}^{19} \mathrm{~N}_{3} \mathrm{O}_{5}^{4}$ & 90.59 & -0.64 & Pro Pro Tyr \\
\hline 159 & 5.570 & $(\mathrm{M}+\mathrm{H})^{+}$ & 434.2276 & 435.2349 & $\mathrm{C}_{20}^{19} \mathrm{H}_{30}^{25} \mathrm{~N}_{6} \mathrm{O}_{5}^{5}$ & 94.18 & 0.48 & Pro Tyr Arg \\
\hline 160 & 7.417 & $(\mathrm{M}+\mathrm{H})^{+}$ & 375.1785 & 376.1861 & $\mathrm{C}_{19} \mathrm{H}_{25} \mathrm{~N}_{3} \mathrm{O}_{5}$ & 93.97 & 2.32 & Pro Tyr Pro \\
\hline 161 & 8.854 & $(\mathrm{M}+\mathrm{Na})^{+}$ & 217.1824 & 240.1717 & $\mathrm{C}_{15} \mathrm{H}_{23} \mathrm{~N}$ & 85.69 & 2.86 & Prolintane \\
\hline 162 & 7.820 & $(\mathrm{M}+\mathrm{H})^{+}$ & 253.1067 & 254.1140 & $\mathrm{C}_{11}^{15} \mathrm{H}_{15} \mathrm{~N}_{3} \mathrm{O}_{4}$ & 85.96 & -1.67 & Pyricarbate \\
\hline 163 & 5.311 & $\left(\mathrm{M}+\mathrm{NH}_{4}\right)^{+}$ & 183.0901 & 201.1237 & $\mathrm{C}_{9} \mathrm{H}_{13} \mathrm{NO}_{3}$ & 90.47 & -3.06 & Racepinephrine \\
\hline 164 & 7.516 & $(\mathrm{M}+\mathrm{H})^{+}$ & 207.0900 & 208.0972 & $\mathrm{C}_{11} \mathrm{H}_{13} \mathrm{NO}_{3}^{3}$ & 85.18 & -2.13 & Rhexifoline \\
\hline 165 & 8.534 & $(\mathrm{M}+\mathrm{H})^{+}$ & 244.1584 & 245.1653 & $\mathrm{C}_{15}^{11} \mathrm{H}_{20} \mathrm{~N}_{2} \mathrm{O}^{3}$ & 88.37 & -3.31 & Rhombifoline \\
\hline 166 & 9.862 & $(\mathrm{M}+\mathrm{H})^{+}$ & 122.1099 & 123.1172 & $\mathrm{C}_{9} \mathrm{H}_{14}^{20}$ & 86.97 & -3.18 & Santene* \\
\hline 167 & 14.655 & $(\mathrm{M}+\mathrm{H})^{+}$ & 299.2829 & 300.2901 & $\mathrm{C}_{18} \mathrm{H}_{37} \mathrm{NO}_{2}$ & 95.29 & -1.68 & Sphingosine \\
\hline 168 & 19.924 & $(\mathrm{M}+\mathrm{H})^{+}$ & 213.2457 & 214.2530 & $\mathrm{C}_{14}^{18} \mathrm{H}_{31} \mathrm{~N}$ & 99.64 & -0.40 & Tetradecylamine* \\
\hline 169 & 19.831 & $(\mathrm{M}+\mathrm{H})^{+}$ & 370.1547 & 371.1620 & $\mathrm{C}_{21}^{14} \mathrm{H}_{26} \mathrm{~N}_{2} \mathrm{~S}_{2}$ & 93.67 & -2.66 & Thioridazine \\
\hline 170 & 4.410 & $(\mathrm{M}+\mathrm{H})^{+}$ & 346.2212 & 347.2284 & $\mathrm{C}_{15} \mathrm{H}_{30} \mathrm{~N}_{4} \mathrm{O}_{5}$ & 80.69 & 1.11 & Thr Val Lys \\
\hline 171 & 7.568 & $(\mathrm{M}+\mathrm{Na})^{+}$ & 493.3240 & 516.3131 & $\mathrm{C}_{28}^{15} \mathrm{H}_{47} \mathrm{NO}_{4}^{4} \mathrm{~S}$ & 87.00 & -2.91 & Tiamulin \\
\hline 172 & 7.166 & $(\mathrm{M}+\mathrm{H})^{+}$ & 253.1068 & 254.1142 & $\mathrm{C}_{12}^{28} \mathrm{H}_{11}^{4 /} \mathrm{N}_{7}^{4}$ & 83.69 & 3.12 & Triamterene \\
\hline 173 & 6.352 & $\left(\mathrm{M}+\mathrm{NH}_{4}\right)^{+}$ & 184.0489 & 202.0827 & $\mathrm{C}_{7} \mathrm{H}_{8} \mathrm{~N}_{2} \mathrm{O}_{4}$ & 98.31 & -2.85 & Trimidox \\
\hline 174 & 9.211 & $(\mathrm{M}+\mathrm{H})^{+}$ & 141.1154 & 142.1228 & $\mathrm{C}_{8} \mathrm{H}_{15}^{8} \mathrm{NO}^{4}$ & 84.62 & -0.41 & Tropine \\
\hline 175 & 7.102 & $(\mathrm{M}+\mathrm{H})^{+}$ & 415.1856 & 416.1930 & $\mathrm{C}_{20} \mathrm{H}_{25} \mathrm{~N}_{5} \mathrm{O}_{5}$ & 83.43 & -0.12 & Trp Asn Pro \\
\hline 176 & 7.010 & $(\mathrm{M}+\mathrm{Na})^{+}$ & 418.1850 & 441.1747 & $\mathrm{C}_{20}^{20} \mathrm{H}_{26} \mathrm{~N}_{4} \mathrm{O}_{6}^{5}$ & 80.39 & 0.59 & Trp Asp Val \\
\hline 177 & 7.210 & $(\mathrm{M}+\mathrm{Na})^{+}$ & 372.1803 & 395.1694 & $\mathrm{C}_{19}^{2} \mathrm{H}_{24} \mathrm{~N}_{4} \mathrm{O}_{4}$ & 81.48 & -1.36 & Trp Pro Ala \\
\hline 178 & 7.817 & $\left(\mathrm{M}+\mathrm{NH}_{4}\right)^{+}$ & 303.1583 & 321.1923 & $\mathrm{C}_{16} \mathrm{H}_{21} \mathrm{~N}_{3} \mathrm{O}_{3}$ & 96.76 & -0.18 & Tryptophyl-Valine \\
\hline 179 & 8.506 & $\left(\mathrm{M}+\mathrm{NH}_{4}\right)^{+}$ & 423.2000 & 441.2342 & $\mathrm{C}_{20}^{10} \mathrm{H}_{29} \mathrm{~N}_{3} \mathrm{O}_{7}$ & 88.19 & 1.20 & Tyr Ile Glu \\
\hline 180 & 8.450 & $\left(\mathrm{M}+\mathrm{NH}_{4}\right)^{+}$ & 396.2002 & 414.2345 & $\mathrm{C}_{18} \mathrm{H}_{28} \mathrm{~N}_{4} \mathrm{O}_{6}$ & 90.22 & 1.70 & Tyr Ser Lys \\
\hline 181 & 6.721 & $(\mathrm{M}+\mathrm{Na})^{+}$ & 516.2543 & 539.2431 & $\mathrm{C}_{25}^{18} \mathrm{H}_{36}^{28} \mathrm{~N}_{6}^{4} \mathrm{O}_{4}^{6} \mathrm{~S}$ & 80.47 & -4.67 & Udenafil \\
\hline 182 & 7.666 & $\left(\mathrm{M}+\mathrm{NH}_{4}\right)^{+}$ & 387.2258 & 405.2597 & $\mathrm{C}_{20} \mathrm{H}_{29} \mathrm{~N}_{5} \mathrm{O}_{3}$ & 80.07 & 3.07 & Urapidil \\
\hline 183 & 6.933 & $(\mathrm{M}+\mathrm{H})^{+}$ & 346.1489 & 347.1562 & $\mathrm{C}_{13}^{20} \mathrm{H}_{22} \mathrm{~N}_{4} \mathrm{O}_{7}^{3}$ & 81.66 & -0.18 & Val Asp Asn \\
\hline 184 & 7.580 & $(\mathrm{M}+\mathrm{H})^{+}$ & 401.2058 & 402.2131 & $\mathrm{C}_{20} \mathrm{H}_{27} \mathrm{~N}_{5} \mathrm{O}_{4}$ & 82.27 & 1.29 & Val His Phe \\
\hline 185 & 7.655 & $(\mathrm{M}+\mathrm{H})^{+}$ & 466.2214 & 467.2286 & $\mathrm{C}_{25} \mathrm{H}_{30} \mathrm{~N}_{4} \mathrm{O}_{5}$ & 93.34 & 0.48 & Val Trp Tyr \\
\hline 186 & 7.189 & $(\mathrm{M}+\mathrm{H})^{+}$ & 254.1385 & 255.1458 & $\mathrm{C}_{11}^{25} \mathrm{H}_{18} \mathrm{~N}_{4}^{4} \mathrm{O}_{3}^{5}$ & 98.02 & -2.38 & Valyl-Histidine \\
\hline 187 & 9.275 & $\left(\mathrm{M}+\mathrm{NH}_{4}\right)^{+}$ & 202.0745 & 220.1084 & $\mathrm{C}_{11}^{11} \mathrm{H}_{10} \mathrm{~N}_{2} \mathrm{O}_{2}^{3}$ & 86.30 & -1.26 & Vasicinone \\
\hline 188 & 8.958 & $(\mathrm{M}+\mathrm{H})^{+}$ & 199.1326 & 200.1399 & $\mathrm{C}_{9} \mathrm{H}_{17} \mathrm{~N}_{3} \mathrm{O}_{2}$ & 82.59 & -2.81 & Vinyl-L-NIO \\
\hline 189 & 5.660 & $\left(\mathrm{M}+\mathrm{NH}_{4}\right)^{+}$ & 157.0856 & 175.1194 & $\mathrm{C}_{6}^{9} \mathrm{H}_{11} \mathrm{~N}_{3} \mathrm{O}_{2}^{2}$ & 98.32 & -3.12 & V-PYRRO/NO \\
\hline 190 & 15.142 & $(\mathrm{M}+\mathrm{H})^{+}$ & 229.2410 & 230.2483 & $\mathrm{C}_{14} \mathrm{H}_{31} \mathrm{NO}^{2}$ & 84.30 & -1.90 & Xestoaminol C* \\
\hline 191 & 17.087 & $(\mathrm{M}+\mathrm{H})^{+}$ & 115.0461 & 116.0534 & $\mathrm{C}_{5} \mathrm{H}_{9} \mathrm{NS}$ & 94.29 & -4.73 & $\begin{array}{l}\text { xi-2,5-Dihydro-2,4- } \\
\text { dimethylthiazole }\end{array}$ \\
\hline 192 & 10.173 & $(\mathrm{M}+\mathrm{H})^{+}$ & 374.0342 & 375.0411 & $\mathrm{C}_{17} \mathrm{H}_{12} \mathrm{C}_{12} \mathrm{~N}_{4} \mathrm{O}_{2}$ & 82.26 & -1.30 & a,4-Dihydroxytriazolam \\
\hline 193 & 14.599 & $\left(\mathrm{M}+\mathrm{NH}_{4}\right)^{+}$ & 222.1991 & 240.2329 & $\mathrm{C}_{15} \mathrm{H}_{26}^{12} \mathrm{O}$ & 97.50 & -3.28 & $\beta$-Caryophyllene Alcohol* \\
\hline
\end{tabular}

*Indicate the metabolites that found similarly from pHMG extract under second evaluation screening. RT=Retention time, $\mathrm{DB}=$ Database, LC-MS=Liquid chromatography-mass spectrometry, QTOF=Quadrupole time-of-flight

macronutrient classification (Figure-2d). The five groups of macronutrients are comprised oligosaccharides, peptides, lipids, nucleosides, and secondary metabolites. There were 192 and 42 metabolites identified from eHMG and $\mathrm{pHMG}$ extracts (Tables-3 and 4), respectively. The differences in the type of metabolites between eHMG and $\mathrm{pHMG}$ extracts have further supported the preference of the type of metabolites toward each extraction method. Among the macronutrients, eHMG extraction method can extract mostly secondary metabolites, followed by peptides, oligosaccharides, lipids, and nucleosides (Figure-2d). The primary metabolites obtained from this study support the finding from the previous proximate analysis of EBN, which protein is the highest composition followed by carbohydrates and lipids [2,36,37].

The presence of secondary metabolites could most probably explain the recuperative and therapeutic effects of EBN. The secondary metabolite with the identity of $\mathrm{O}^{2}$-vinyl 1-(pyrrolidin-1-yl)diazen-1-ium1,2-diolate (V-PYRRO/nitric oxide [NO]) was found in eHMG extract (Table-3). This secondary metabolite acts as NO donor and delivers NO specifically after metabolism by cytochrome P450 in hepatocytes without affecting the NO-sensitive tissues as well as systolic blood pressure [38]. The in vivo study done by $\mathrm{Li}$ et al. [39] showed that V-PYRRO/NO is able to protect the hindrance to renal congestion and lipid peroxidation from acetaminophen-induced nephrotoxicity in mice. In addition, V-PYRRO/NO can protect against high-fat diet (HFD)-induced liver steatosis and insulin resistance without affecting the mitochondria biogenesis [40]. Interestingly, Zhang et al. [41] showed that EBN could prevent HFD-induced insulin resistance by regulating the transcriptional changes in insulin signaling genes. Hence, the presence of V-PYRRO/NO in EBN may explain the protective effect of EBN against the HFDinduced damages. In short, from this study, it is believed that the study on secondary metabolites profiling in EBN in the future is crucial and not to be neglected.

A polysaccharide with an identity of chondroitin was identified from the first screening of eHMG extract 
Table-4: Information of the metabolites in PHMG extract. The metabolites are identified by QTOF LC-MS with second screening evaluation.

\begin{tabular}{|c|c|c|c|c|c|c|c|c|}
\hline Number & $\begin{array}{c}\text { RT } \\
(\mathbf{m i n})\end{array}$ & Ion & Mass & $\mathbf{m} / \mathbf{z}$ & $\begin{array}{l}\text { Molecular } \\
\text { formula }\end{array}$ & Score & $\begin{array}{c}\text { DB differences } \\
(\mathrm{ppm})\end{array}$ & $\begin{array}{l}\text { Putatively identified } \\
\text { metabolites }\end{array}$ \\
\hline 1 & 19.793 & $(\mathrm{M}+\mathrm{Na})^{+}$ & 484.3396 & 507.3290 & $\mathrm{C}_{27} \mathrm{H}_{48} \mathrm{O}_{7}$ & 96.12 & 0.87 & $\begin{array}{l}\text { (25S)-5alpha-cholestan-3beta,4 } \\
\text { beta,6alpha,8beta,15alpha,16b } \\
\text { eta,26-heptol }\end{array}$ \\
\hline 2 & 3.780 & $(\mathrm{M}+\mathrm{H})^{+}$ & 99.0322 & 100.0394 & $\mathrm{C}_{4} \mathrm{H}_{5} \mathrm{NO}_{2}$ & 86.24 & -1.50 & (R)-Dihydromaleimide \\
\hline 3 & 14.655 & $\left(\mathrm{M}+\mathrm{NH}_{4}\right)^{+}$ & 168.1881 & 186.2219 & & 98.94 & -2.06 & 1-Dodecene* \\
\hline 4 & 19.883 & $(\mathrm{M}+\mathrm{H})^{+}$ & 241.2768 & 242.2840 & ${ }_{55}^{4} \mathrm{~N}$ & 86.03 & 0.67 & 1-Hexadecylamine \\
\hline 5 & 17.082 & $(\mathrm{M}+\mathrm{H})^{+}$ & 203.0807 & 204.0878 & $\mathrm{C}_{9} \mathrm{H}_{17} \mathrm{NS}_{2}$ & 93.79 & -2.07 & $\begin{array}{l}\text { 1-Isothiocyanato-7-(methylthio) } \\
\text { heptane* }\end{array}$ \\
\hline 6 & 15.586 & $(\mathrm{M}+\mathrm{H})^{+}$ & 115.0457 & 116.0530 & $\mathrm{C}_{5} \mathrm{H}_{9} \mathrm{NS}$ & 99.79 & -1.42 & 1-Isothiocyanatobutane* \\
\hline 7 & 19.896 & $\left(\mathrm{M}+\mathrm{NH}_{4}\right)^{+}$ & 392.4383 & 410.4722 & & 98.76 & -0.37 & 1-Octacosene* \\
\hline 8 & 17.084 & $(\mathrm{M}+\mathrm{H})^{+}$ & 171.1085 & 172.1157 & NS & 96.61 & -1.92 & $\begin{array}{l}\text { 2,5-Dihydro- } 4,5 \text {-dimethyl-2-(1- } \\
\text { methylpropyl)thiazole* }\end{array}$ \\
\hline 9 & 18.737 & $(\mathrm{M}+\mathrm{H})^{+}$ & 170.1303 & 171.1375 & ${ }_{18} \mathrm{O}_{2}$ & 84.98 & 2.15 & $\begin{array}{l}\text { 2,6-Dimethyl-3,7-octadiene-2,6- } \\
\text { diol }\end{array}$ \\
\hline 10 & 7.676 & $\left(\mathrm{M}+\mathrm{NH}_{4}\right)^{+}$ & 256.1315 & 274.1653 & $\mathrm{C}_{13}$ & 98.83 & -1.55 & $\begin{array}{l}\text { 2-[4-(3-Hydroxypropyl)- } \\
\text { 2-methoxyphenoxy]-1,3- } \\
\text { propanediol }\end{array}$ \\
\hline 11 & 7.678 & $(\mathrm{M}+\mathrm{H})^{+}$ & 113.0845 & 114.0918 & $\mathrm{C}_{6} \mathrm{H}_{11} \mathrm{NO}$ & 98.61 & -3.80 & 2-Acetylpyrrolidine* \\
\hline 12 & 14.532 & $(\mathrm{M}+\mathrm{H})^{+}$ & 105.0790 & 106.0863 & $\mathrm{NO}_{2}$ & 84.69 & -0.41 & $\begin{array}{l}\text { 2-Amino-2-methyl-1,3- } \\
\text { propanediol }\end{array}$ \\
\hline 13 & 9.461 & $(\mathrm{M}+\mathrm{H})^{+}$ & 144.0422 & 145.0493 & $\mathrm{C}_{6} \mathrm{r}$ & 95.91 & 0.31 & $\begin{array}{l}\text { 2-Hydroxy-2-(hydroxymethyl)- } \\
\text { 2H-pyran-3(6H)-one }\end{array}$ \\
\hline 14 & 9.859 & $(\mathrm{M}+\mathrm{H})^{+}$ & 101.0844 & 102.0916 & NO & 96.89 & -3.15 & 2-methylbutanal oxime \\
\hline 15 & 8.921 & $(\mathrm{M}+\mathrm{H})^{+}$ & 209.1423 & 210.1496 & $\mathrm{C}_{12} \mathrm{H}_{19} \mathrm{NO}_{2}$ & 84.38 & -3.50 & 3,4-dimethoxymethamphetamine \\
\hline 16 & 19.503 & $\left(\mathrm{M}+\mathrm{NH}_{4}\right)^{+}$ & 135.0564 & 153.0902 & & 82.59 & -4.21 & $\begin{array}{l}\text { 4-(Hydroxymethyl) } \\
\text { benzenediazonium }(1+)\end{array}$ \\
\hline 17 & 7.839 & $(\mathrm{M}+\mathrm{H})^{+}$ & 125.0839 & 126.0912 & NO & 87.69 & 1.30 & 4-Ethyl-2,5-dimethyloxazole \\
\hline 18 & 6.992 & $(\mathrm{M}+\mathrm{H})^{+}$ & 129.0430 & 130.0503 & $\mathrm{C}_{5} \mathrm{H}_{7} \mathrm{NO}_{3}$ & 82.11 & -3.43 & 4-Oxoproline* \\
\hline 19 & 8.905 & $(\mathrm{M}+\mathrm{H})^{+}$ & 153.1156 & 154.1227 & & 95.68 & -1.34 & 5-Butyl-2-ethyloxazole \\
\hline 20 & 7.182 & $\left(\mathrm{M}+\mathrm{NH}_{4}\right)^{+}$ & 165.1155 & 183.1491 & ${ }_{5} \mathrm{NO}$ & 82.05 & -0.72 & $\begin{array}{l}\text { 5-Methyl-2-(1-pyrrolidinyl)-2- } \\
\text { cyclopenten-1-one* }\end{array}$ \\
\hline 21 & 16.002 & $\left(\mathrm{M}+\mathrm{NH}_{4}\right)^{+}$ & 250.2302 & 268.2640 & $\mathrm{C}_{1}$ & 85.38 & -2.25 & $\begin{array}{l}\text { 9S,10R-Epoxy-3Z,6Z- } \\
\text { octadecadiene* }\end{array}$ \\
\hline 22 & 19.912 & $\left(\mathrm{M}+\mathrm{NH}_{4}\right)^{+}$ & 364.4070 & 382.4409 & & 99.67 & -0.34 & 9Z-Hexacosene* \\
\hline 23 & 5.021 & $(\mathrm{M}+\mathrm{H})^{+}$ & 135.0550 & 136.0622 & & 86.53 & -3.53 & Adenine* \\
\hline 24 & 5.761 & $(\mathrm{M}+\mathrm{H})^{+}$ & 226.1066 & 227.1139 & $\mathrm{~N}_{4} \mathrm{O}_{3}$ & 92.45 & 0.04 & Alanyl-Histidine* \\
\hline 25 & 18.616 & $(\mathrm{M}+\mathrm{H})^{+}$ & 142.0013 & 143.0084 & $\mathrm{~N}_{2} \mathrm{O}_{4}^{3}$ & 80.18 & 0.76 & Alloxan \\
\hline 26 & 5.765 & $(\mathrm{M}+\mathrm{H})^{+}$ & 109.0641 & 110.0713 & & 83.73 & -1.28 & Brunfelsamidine* \\
\hline 27 & 7.804 & $\left(\mathrm{M}+\mathrm{NH}_{4}\right)^{+}$ & 922.4758 & 940.5092 & $\mathrm{O}_{20}$ & 95.22 & 1.67 & Capsianoside VI \\
\hline 28 & 19.762 & $\left(\mathrm{M}+\mathrm{NH}_{4}\right)^{+}$ & 747.4789 & 765.5129 & ${ }_{9}^{4} \mathrm{NO}_{13}$ & 85.36 & -2.63 & Clarithromycin \\
\hline 29 & 17.082 & $(\mathrm{M}+\mathrm{H})^{+}$ & 127.1363 & 128.1435 & & 86.72 & -1.61 & Coniine* \\
\hline 30 & 19.949 & $\left(\mathrm{M}+\mathrm{NH}_{4}\right)^{+}$ & 703.4523 & 721.4865 & ${ }_{5} \mathrm{NO}_{12}$ & 80.50 & -2.29 & Erythromycin D \\
\hline 31 & 13.733 & $(\mathrm{M}+\mathrm{Na})^{+}$ & 270.1830 & 293.1723 & & 86.19 & 0.32 & Ethylene brassylate \\
\hline 32 & 19.854 & $\left(\mathrm{M}+\mathrm{NH}_{4}\right)^{+}$ & 240.2452 & 258.2791 & & 85.17 & 0.68 & hexadeca-9-en-1-ol \\
\hline 33 & 19.781 & $(\mathrm{M}+\mathrm{H})^{+}$ & 101.1201 & 102.1274 & & 87.12 & 3.51 & Hexylamine* \\
\hline 34 & 18.616 & $(\mathrm{M}+\mathrm{H})^{+}$ & 298.1537 & 299.1607 & $\mathrm{C}_{15} \mathrm{H}_{18} \mathrm{~N}_{6} \mathrm{O}$ & 83.14 & 1.86 & Iso-Olomoucine* \\
\hline 35 & 8.676 & $\left(\mathrm{M}+\mathrm{NH}_{4}\right)^{+}$ & 268.1312 & 286.1649 & & 80.46 & -0.63 & Kamahine C* \\
\hline 36 & 3.375 & $(\mathrm{M}+\mathrm{Na})^{+}$ & 383.1426 & 406.1318 & ${ }_{25} \mathrm{NO}_{11}$ & 94.48 & 0.36 & Lacto-N-biose I* \\
\hline 37 & 19.300 & $(\mathrm{M}+\mathrm{H})^{+}$ & 129.1511 & 130.1585 & & 83.91 & 4.67 & Octylamine* \\
\hline 38 & 14.588 & $(\mathrm{M}+\mathrm{H})^{+}$ & 255.2565 & 256.2637 & $\mathrm{H}_{33} \mathrm{NO}$ & 98.75 & -1.08 & Palmitic amide* \\
\hline 39 & 9.858 & $(\mathrm{M}+\mathrm{H})^{+}$ & 122.1096 & 123.1169 & & 85.95 & -0.42 & Santene* \\
\hline 40 & 19.911 & $(\mathrm{M}+\mathrm{H})^{+}$ & 213.2457 & 214.2530 & & 98.18 & -0.32 & Tetradecylamine* \\
\hline 41 & 19.838 & $(\mathrm{M}+\mathrm{H})^{+}$ & 229.2406 & 230.2478 & $\mathrm{C}_{14}^{14} \mathrm{H}_{31} \mathrm{NO}$ & 97.56 & 0.01 & Xestoaminol C* \\
\hline 42 & 14.568 & $\left(\mathrm{M}+\mathrm{NH}_{4}\right)^{+}$ & 222.1988 & 240.2326 & $\mathrm{C}_{15} \mathrm{H}_{26} \mathrm{O}$ & 98.81 & -2.08 & $\beta$-Caryophyllene Alcohol* \\
\hline
\end{tabular}

*Indicate the metabolites that found similarly from eHMG extract under second evaluation screening. RT=Retention time, $\mathrm{DB}=$ Database, LC-MS=Liquid chromatography-mass spectrometry, QTOF=Quadrupole time-of-flight

(Table-1), in which the discovery of water-soluble chondroitin is similar to the finding of Nakagawa et al. in EBN [42]. Chondroitin is a glycosaminoglycan that acts as a chondroprotective agent for the treatment of OA. OA is the lesion of articular cartilage caused by trauma. Since chondroitin is an essential proteoglycan in cartilage, it acts on OA by stimulates the cartilage repair through enhancing the production of the extracellular matrix of cartilage. Besides, chondroitin helps to maintain the viscosity of the synovial fluid to lubricate the joint and therefore reducing the pain of the patient. Furthermore, chondroitin suppresses the inflammatory cytokines such as interleukin- $1 \beta$ that induce the release of matrix metalloproteinases and 
aggrecanases which cause the degradation of the cartilage $[43,44]$. In an in vitro study done by Chua et al. on the effects of EBN to OA [17], the authors reported that EBN can protect articular cartilage from further deterioration by reducing inflammation and enzymatic lesions process and enhancing the cartilage formation simultaneously. Therefore, the effects of EBN on OA might be contributed by chondroitin.

\section{Conclusion}

There was no single extraction method could provide optimal conditions in extracting all the metabolites from EBN. Therefore, complementary extraction methods should be used in parallel when broader metabolite profiles are required. eHMG extraction method was selected as the ideal extraction method for untargeted profiling the type of polar metabolites in EBN. This is because the number and the type of metabolites detected are the highest in eHMG extracts among the four evaluated extraction methods. Furthermore, the presence of key metabolites of sialic acid has further defined the suitability of eHMG extraction method. Therefore, the findings in this study could offer great potential for enhancement in the industrial EBN extraction process and hence improve the overall EBN yield and bioactivities. Nevertheless, the validation of the structure elucidation and functional assays of interesting metabolites shall be carried out in the future.

\section{Authors' Contributions}

YML conceived the study design. SRT conducted all the designed experiments, data processing, and analysis. THL contributed to the sample collection and performed the in-house extraction method (eHMG and pHMG) for the study. SRT prepared the manuscript with critical feedback from the coauthors. THL, SKC, and YML supervised the study and provided input and advice in the project. All authors have read and approved the final manuscript.

\section{Acknowledgments}

This project is funded by the Ministry of Agriculture and Agro-Based Industry (MOA), Malaysia. Besides, UTAR also provided financial support through UTAR Research Fund (UTARRF) with vote number of IPSR/ RMC/UTARRF/2016-C2/L12. The authors show the gratitude to all the ranchers of swiftlet's house for providing the EBN samples in this study, as well as the University Laboratory Management Centre (PPMU) in UTM for technical support.

\section{Competing Interests} interests.

The authors declare that they have no competing

\section{Publisher's Note}

Veterinary World remains neutral with regard to jurisdictional claims in published institutional affiliation.

\section{References}

1. Ma, F. and Liu, D. (2012) Sketch of the edible bird's nest and it's important bioactivities. Food Res. Int., 48(2): 559-567.

2. Marcone, M.F. (2005) Characterization of the edible bird's nest the "Caviar of the East". Food Res. Int., 38(10): $1125-1134$.

3. Norhayati, M.K., Azman, O. and Wan Nazaimoon, W.M. (2010) Preliminary study of the nutritional content of Malaysian edible bird's nest. Malays. J. Nutr., 16(3): 389-396.

4. Lee, T.H., Wani, W.A., Tan, T.T.E., Adnan, N.A., Yong, L.L. and Aziz, R.A. (2015) Investigations into the physicochemical, biochemical and antibacterial properties of edible bird's nest. J. Chem. Pharm. Res., 7(7): 228-247.

5. Wang, C.C. (1921) The composition of Chinese edible birds' nests and the nature of their proteins. J. Biol. Chem., 49: 429-439.

6. Seow, E.K., Ibrahim, B., Muhammad, S.A., Lee, L.H. and Cheng, L.H. (2016) Differentiation between house and cave edible bird's nests by chemometric analysis of amino acid composition data. LWT Food Sci. Technol., 65: 428-435.

7. Wong, R.S.Y. (2013) Edible bird's nest: Food or medicine? Chin. J. Integr. Med., 19(9): 643-649.

8. Rashed, A.A. and Wan Nazaimoon, W.M. (2010) Effect of edible bird's nest on Caco-2 cell proliferation. J. Food Technol., 8(3): 126-130.

9. Guo, C.T., Takahashi, T., Bukawa, W., Takahashi, N., Yagi, H., Kato, K., Hindari, K.I.P., Miyamoto, D., Suzuki, T. and Suzuki, Y. (2006) Edible bird's nest extract inhibits influenza virus infection. Antiviral Res., 70(3): 140-146.

10. Matsukawa, N., Matsumoto, M., Bukawa, W., Chiji, H., Nakayame, K., Hara, H. and Tsukahara, T. (2011) Improvement of bone strength and dermal thickness due to dietary edible bird's nest extract in ovariectomized rats. Biosci. Biotechnol. Biochem., 75(3): 590-592.

11. Abidin, F.Z., Chua, K.H., Ng, S.L., Ramli, E.S.M., Lee, T.H. and Ghafar, N.A. (2011) Effects of edible bird's nest (EBN) on cultured rabbit corneal keratocytes. BMC Complement Altern. Med., 11(1): 94.

12. Land, M.F. and Fernald, R.D. (1992) The evolution of eyes. Annu. Rev. Neurosci., 15: 1-29.

13. Hou, Z., Imam, M.U., Ismail, M., Azmi, N.H., Ismail, N., Ideris, A. and Mahmud, R. (2015) Lactoferrin and ovotransferrin contribute toward antioxidative effects of edible bird's nest against hydrogen peroxide-induced oxidative stress in human SH-SY5Y cells. Biosci. Biotechnol. Biochem., 79(10): 1570-1578.

14. Yida, Z., Imam, M.U. and Ismail, M. (2014) In vitro bioaccessibility and antioxidant properties of edible bird's nest following simulated human gastro-intestinal digestion. BMC Complement Altern. Med., 14: 468.

15. Roh, K., Lee, J., Kim, Y., Park, J., Kim, J., Lee, J. and Park, D. (2011) Mechanisms of edible bird's nest extract-induced proliferation of human adipose-derived stem cells. Evid. Based Complement Alternat. Med., 2012: Article ID 797520.

16. Kong, Y.C., Keung, W.M., Yip, T.T., Ko, K.M., Tsao, S.W. and Ng, M.H. (1987) Evidence that epidermal growth factor is present in swiftlet's (Collocalia) nest. Comp. Biochem. Physiol., 878(2): 221-226.

17. Chua, K.H., Lee, T.H., Nagandran, K., Yahaya, N.H.M., Lee, C.T., Tan, E.T.T. and Aziz, R.A. (2013) Edible Bird's nest extract as a chondroprotective agent for human chondrocytes isolated from osteoarthritic knee: In vitro study. BMC Complement. Altern. Med., 13(1): 19.

18. Aswir, A.R. and Wan Nazaimoon, W.M. (2011) Effect of edible bird's nest on cell proliferation and tumor necrosis factor-alpha (TNF- $\alpha)$ release in vitro. Int. Food Res. J., 18(3): 1123-1127.

19. Liu, F., Gan, P.P., Wu, H., Woo, W.S., Ong, E.S. and Li, S.F. 
(2012) A combination of metabolomics and metallomics studies of urine and serum from hypercholesterolaemic rats after berberine injection. Anal. Bioanal. Chem., 403(3): 847-856.

20. Theodoridis, G., Gika, H.I., Franceschi, P., Caputi, L., Arapitsas, P., Scholz, M., Masuero, D., Wehrens, R., Vrhovsek, U. and Mattivi, F. (2011) LC-MS based global metabolite profiling of grapes: Solvent extraction protocol optimisation. Metabolomics, 8(2): 175-185.

21. Pongsuwan, W., Fukusaki. E., Bamba, T., Yonetani, T., Yamahara, T. and Kobayashi, A. (2007) Prediction of Japanese green tea ranking by gas chromatography/mass spectrometry-based hydrophilic metabolite fingerprinting. J. Agric. Food Chem., 55(2): 231-236.

22. Chua, Y.G., Bloodworth, B.C., Leong, L.P. and Li, S.F.Y. (2014) Metabolite profiling of edible bird's nest using gas chromatography/mass spectrometry and liquid chromatography/mass spectrometry. Rapid Commun. Mass Spectrom., 28(12): 1387-1400.

23. Oda, M., Ohta, S., Suga, T. and Aoki, T. (1998) Study on food components : The structure of N-linked asialo carbohydrate from the edible bird's nest built by Collocalia fuciphaga. J. Agric. Food Chem., 46: 3047-3053.

24. Goh, D.L.M., Chua, K.Y., Chew, F.T., Seow, T.K., Ou, K.L., Yi, F.C. and Lee, B.W. (2001) Immunochemical characterization of edible bird's nest allergens. J. Allergy Clin. Immunol., 107(6): 1082-1088.

25. Ho, Y.S. (2016) Elucidation of Nutritional and Metabolite Profiles and Regenerative Effect of Edible Bird's Nest on Neuron Cells. Master Dissertation. Universiti Tunku Abdul Rahman, Malaysia.

26. Xiao, J.F., Zhou, B. and Ressom, H.W. (2012) Metabolite identification and quantitation in LC-MS/MS-based metabolomics. TRAC-Trend Anal. Chem., 32: 1-14.

27. Sandra, K., Pereira, A.S., Vanhoenacker, G., David, F. and Sandra, P. (2010). Comprehensive blood plasma lipidomics by liquid chromatography/quadrupole time-of-flight mass spectrometry. J. Chromatogr. A, 1217(25): 4087-4099.

28. Cruickshank-Quinn, C., Quinn, K.D., Powell, R., Yang, Y., Armstrong, M., Mahaffey, S., Reisdorph, R. and Reisdorph, N. (2014) Multi-step preparation technique to recover multiple metabolite compound classes for in-depth and informative metabolomics analysis. J. Vis. Exp., 89(89): e51670.

29. Sellick, C.A., Knight, D., Croxford, A.S., Maqsood, A.R., Stephens, G.M., Goodacre, R. and Dickson, A.J. (2010) Evaluation of extraction processes for intracellular metabolite profiling of mammalian cells: Matching extraction approaches to cell type and metabolite targets. Metabolomics, 6(3): 427-438.

30. Xu, Y.Q., Ji, W.B., Yu, P., Chen, J.X., Wang, F. and Yin, J.F. (2017) Effect of extraction methods on the chemical components and taste quality of green tea extract. Food Chem., 248: 146-154.

31. Mtewa, A.G., Deyno, S., Kasali, F.M., Annu, A. and Sesaazi, D.C. (2018) General extraction, isolation and characterization techniques in drug discovery: A review. Int. J. Sci. Basic Appl. Res., 38(1): 10-24.

32. Gupta, A., Naraniwal, M. and Kothari, V. (2012) Modern extraction methods for preparation of bioactive plant extracts. Int. J. Appl. Nat. Sci., 1(1): 8-26.

33. Quek, M.C., Chin, N.L., Yusof, Y.A., Law, C.L. and Tan, S.W. (2018) Characterization of edible bird's nest of different production, species and geographical origins using nutritional composition, physicochemical properties and antioxidant activities. Food Res. Int., 109: 35-43.

34. Chan, G.K., Zheng, K.Y., Zhu, K.Y., Dong, T.T. and Tsim, K.W. (2013) Determination of free $\mathrm{N}$-acetylneuraminic acid in edible bird nest: A development of chemical marker for quality control. J. Ethnobiol. Tradit. Med., 120: 620-628.

35. Marni, S., Marzura, M.R., Norzela, A.M., Khairunnisak, M., Bing, C.H. and Eddy, A.A. (2014) Preliminary study on free sialic acid content of edible bird nest from Johor and Kelantan. Malays. J. Vet. Res., 5(1): 9-14.

36. Saengkrajang, W., Matan, M. and Matan, N. (2013) Nutritional composition of the farmed edible bird's nest (Collocalia fuciphaga) in Thailand. J. Food Compos. Anal., 31(1): 41-45.

37. Huda, M.Z.N., Zuki, A.B.Z., Azhar, K., Goh, Y.M. and Suhaimi, H. (2008) Proximate, elemental and fatty acid analysis of pre-processed edible bird's nest (Aerodramus fuciphagus): A comparison between regions and type of nest. J. Food Technol., 6(1): 39-44.

38. Saavedra, J.E., Billiar, T.R., Williams, D.L., Kim, Y.M., Watkins, S.C. and Keefer, L.K. (1997) Targeting nitric oxide (NO) delivery in vivo. Design of a liver-selective NO donor prodrug that blocks tumor necrosis factor- $\alpha$-induced apoptosis and toxicity in the liver. J. Med. Chem., 40(13): 1947-1954

39. Li, C., Liu, J., Saavedra, J.E., Keefer, L.K. and Waalkes, M.P. (2003) The nitric oxide donor, V-PYRRO/NO, protects against acetaminophen-induced nephrotoxicity in mice. Toxicology, 189(3): 173-180.

40. Maslak, E., Zabielski, P., Kochan, K., Kus, K., Jasztal, A., Sitek, B., Proniewski, B., Wojcik, T., Gula, K., Kij, A., Walczak, M., Baranska, M., Chabowski, A., Holland. R.J., Saavedra. J.E., Keefer L.K. and Chlopicki, S. (2015) The liver-selective NO donor, V-PYRRO/NO, protects against liver steatosis and improves postprandial glucose tolerance in mice fed high-fat diet. Biochem. Pharmacol., 93(3): 389-400.

41. Zhang, Y., Mustapha, U.I., Maznah, I., Oii, D.J., Nadarajan, S., Nur Hanisah, A., Norsharina, I., Chan, K.W., Hou, Z.P. and Norhayati, Y. (2015) Edible bird's nest prevents high fat diet-induced insulin resistance in rats. $J$. Diabetes Res., 2015: 11.

42. Nakagawa, H., Hama, Y., Sumi, T., Li, S.C., Maskos, K., Kalayanamitra, K., Mizumoto, S., Sugahara, K. and Li, Y.T. (2007) Occurrence of a nonsulfated chondroitin proteoglycan in the dried saliva of Collocalia swiftlets (edible bird'snest). Glycobiology, 17(2): 157-164.

43. Vasiliadis, H.S. and Tsikopoulos, K. (2017) Glucosamine and chondroitin for the treatment of osteoarthritis. World $J$. Orthop., 8(1): 1-11.

44. Huskisson, E.C. (2008) Glucosamine and chondroitin for osteoarthritis. J. Int. Med. Res., 36(6): 1-19. 